\title{
Homological Properties of the Homology Algebra of the Koszul Complex of a Local Ring. Examples and Questions.
}

\author{
Jan-Erik Roos \\ Department of Mathematics \\ Stockholm University \\ SE-106 91 Stockholm, SWEDEN \\ e-mail: jeroos@math.su.se \\ Dedicated to Jörgen Backelin at his $65^{\text {th }}$ birthday.
}

December 30, 2015

\begin{abstract}
.
Let $R$ be a local commutative noetherian ring and $H K R$ the homology ring of the corresponding Koszul complex. We study the homological properties of $H K R$ in particular the so-called Avramov spectral sequence. When the embedding dimension of $R$ is four and when $R$ can be presented with quadratic relations we have found 102 cases where this spectral sequence degenerates and only three cases where it does not degenerate. We also determine completely the Hilbert series of the bigraded Tor of these $H K R$ in tables A-D of section 5. We also study some higher embedding dimensions. Among the methods used are the programme BERGMAN by Jörgen Backelin et al, the Macaulay2-package DGAlgebras by Frank Moore, combined with results by Govorov, Clas Löfwall, Victor Ufnarovski and others.
\end{abstract}

Mathematics Subject Classification (2010): Primary 13Dxx, 16Dxx, 68W30; Secondary 16S37, 55Txx

Keywords. local ring, Koszul complex, Gorenstein rings, Yoneda Ext-algebra, Hilbert series, Macaulay2, programme BERGMAN

\section{Introduction and Main Theorem}

Let $(R, m, k)$ be a local commutative noetherian ring with maximal ideal $m$ and residue field $k=R / m$. Let $\mathbf{x}=\left(x_{1}, x_{2}, \ldots, x_{n}\right)$ be a minimal set of generators of the maximal ideal $m$. The Koszul complex of $R$ is the exterior algebra $\oplus_{i=0}^{n} \Lambda^{i} R^{n}$ of a free $R$-module of rank n equipped with the differential:

$$
d\left(T_{j_{1}} \wedge \ldots \wedge T_{j_{i}}\right)=\sum_{l=1}^{i}(-1)^{l+1} x_{j_{l}} T_{j_{1}} \wedge \ldots \wedge \widehat{T}_{j_{l}} \wedge \ldots \wedge T_{j_{i}}
$$

It will be denoted by $K(\mathbf{x}, R)$ or $K R$ since it is essentially independent of $\mathbf{x}$. It is a differential graded algebra and its homology algebra $H K R$ is a skew-commutative graded algebra over $k$. In the case that $R$ can be represented as a quotient of a regular local ring $(\tilde{R}, \tilde{m})$ as $R=\tilde{R} / a$, where $a \subset \tilde{m}^{2}$ (passing to a completion of $R$ we can always assume that this is the case for the problems we are studying), we have that $H K R$ is isomorphic to a Tor-algebra:

$$
H K R \simeq \operatorname{Tor}_{*}^{\tilde{R}}(\tilde{R} / a, k)
$$

This algebra $H K R$ has been studied in various special cases by many authors: it is an exterior algebra if and only if $R$ is a local complete intersection; it is a Poincaré duality algebra it and only if $R$ is a Gorenstein local ring [A-G] and if $R$ is a Golod ring then the square of the augmentation ideal of $H K R$ is 0 (the converse is however not true, even for rings with monomial relations [KAT]). But the general structure of $H K R$ (and in particular its homological properties) can be very complicated, even if $R$ is a Koszul ring as we will see below. The aim of the present paper is to combine different methods towards studying $H K R$. We will illustrate our methods on very precise examples, thereby discovering some new unexpected phenomena. We note that $H K R$ is graded, and one of our aims is to determine the double series

$$
\Phi_{R}(x, y)=\sum_{p \geq 0, q \geq 0}\left|\operatorname{Tor}_{p, q}^{H K R}(k, k)\right| x^{p} y^{q}
$$


(where $|V|$ denotes the dimension of a $k$-vector space $V$ ) for most (probably essentially all) quadratic rings $R$ of embedding dimension 4: Let $P_{R}(z)=\sum_{n \geq 0}\left|\operatorname{Tor}_{n}^{R}(k, k)\right| z^{n}$. We will see that with the exception of three explicit cases we have $\Phi_{R}(z, z)=P_{R}(z) /(1+z)^{4}$. This last equality can be expressed by saying that the so-called Avramov spectral sequence degenerates. Recall that this spectral sequence [AV1, formula (6.2.1)], is as follows for any local ring $R$

$$
E_{n}^{2}=\bigoplus_{p+q=n} E_{p, q}^{2}=\bigoplus_{p+q=n} \operatorname{Tor}_{p, q}^{H K R}(k, k) \Longrightarrow \operatorname{Tor}^{R}(k, k) \otimes_{K R \otimes k} k
$$

In particular there is a coefficientwise inequality $\ll$ of formal power series

$$
\frac{P_{R}(z)}{(1+z)^{m}} \ll \Phi_{R}(z, z)
$$

where $m$ is the embedding dimension of $R$. Of the three exceptional cases in embedding dimension 4, two are Koszul rings with Hilbert series $1+4 z+3 z^{2}$ and $\left(1+3 t-2 t^{3}\right) /(1-t)$ respectively. In these two cases $H K R$ is far from being a Koszul ring, indeed for the first case the bigraded Ext-algebra $\operatorname{Ext}_{H K R}^{*}(k, k)$ has also generators in bidegrees $(3,8)$ and $(3,9)$. Since there are other Koszul rings having the same series $1+4 z+3 z^{2}$ without this strange behaviour, we have here isolated new homological invariants even for Koszul rings. Let us recall that the Avramov spectral sequence always degenerates in embedding dimension $\leq 3$ and for higher embedding dimensions a consequence of it can be expressed by saing that $H K R$ and its so-called Massey products determine $P_{R}(z)$. The converse is not true and the present paper shows that by studying the homology of $H K R$ directly we sometimes obtain interesting invariants for $R$. Our methods also show e.g. that for the ring $k[x, y, z, u] /\left(x^{2}, x y, y z, z u, u^{2}\right)$ the Avramov spectral sequence does degenerate, despite the fact that there is no differential algebra structure on the minimal resolution of the ideal $\left(x^{2}, x y, y z, z u, u^{2}\right)$ over $k[x, y, z, u]$, thereby answering a question by Avramov in [AV1] in the negative. We will use a very useful package DGAlgebras (written by Frank Moore [MOO]) which runs under Macaulay2. This package gives in particular an explicit presentation with generators and relations for $H K R$, when you read in the ring $R$. This presentation will then be analyzed, using results by Clas Löfwall [L1] (the cube of the augmentation ideal of $H K R$ is often 0), Jörgen Backelin et al [BA] (the programme BERGMAN), Victor Ufnarovskij (how to use BERGMAN for determining Hilbert series of noncommutative graded algebras, where some of the generators have degrees > 1) and others. In the tables of Appendix 1 we have described 83 different cases of the double series

$$
P_{R}(x, y)=\sum_{p \geq 0, q \geq 0}\left|\operatorname{Tor}_{p, q}^{R}(k, k)\right| x^{p} y^{q}
$$

when $R$ is a quotient of a polynomial ring $k[x, y, z, u]$ with an ideal generated by quadratic forms in $(x, y, z, u)$. (Note that the previous $P_{R}(z)=P_{R}(z, 1)$.) We have probably obtained all different such cases, but inside each case there are sometimes variations due to the behavior of $H K R$, described in tables A,B,C,D of section 5 below. (this is like the periodic table of the elements, where we also have isotopes). Examples of this are the cases 46, 63 and $\mathbf{7 1}$. More precisely here is our

MAIN THEOREM Let $R=k[x, y, z, u] /\left(f_{1}, f_{2}, \ldots, f_{t}\right)$, where $k$ is a field of characteristic 0 and the $f_{i}$ :s are quadratic forms in the variables $x, y, z, u$ and $P_{R}(x, y)$ is given by (2) above. Then there are 83 different cases known for the $P_{R}(x, y)$ (they are given in Appendix 1). Inside each of these cases $P_{R}(x, y)$ there are sometimes "isotopes" having different homological properties of the homology algebra $H K R$ of the Koszul complex. In total there are 22 such extra "isotopes" known, the most extreme case of $P_{R}(x, y)$ is case 71 which is a Koszul algebra, but which has 4 extra isotopes. With the exception of three of these $105=83+22$ cases we have that $\Phi_{R}(z, z)=P_{R}(z) /(1+z)^{4}$ (i.e. the so-called Avramov spectral sequence degenerates). The three exceptional cases being

a) Case $63 n$ ne (an isotope of Case 63$)$ given by the ideal $\left(x^{2}, x y, x z+u^{2}, x u, y^{2}+z^{2}, z u\right)$ and where

$$
(1+z)^{4} / P_{R_{63 n e}}(z)-1 / \Phi_{63 n e}(z, z)=z^{7}(1+z) /\left(1-z+z^{2}\right)
$$

b) Case 71v16 (an isotope of Case 71) given by the ideal $\left(x z+u^{2}, x y, x u, x^{2}, y^{2}+z^{2}, z u, y z\right)$ and where

$$
(1+z)^{4} / P_{R_{71 v 16}}(z)-1 / \Phi_{R_{71 v 16}}(z, z)=z^{7}(1+z) /\left(1-z+z^{2}\right)
$$


c) Case 60va given by the ideal $\left(x^{2}+y z+u^{2}, x y, z u, z^{2}, x z+y u, x u\right)$ and where

$$
(1+z)^{4} / P_{R_{60 v a}}(z)-1 / \Phi_{R_{60 v a}}(z, z)=z^{7}(1+z)\left(1-3 z^{2}-z^{3}\right) /\left(1-z+z^{2}\right)
$$

Note that a) and b) are Koszul rings. We have also calculated the two-variable $\Phi_{R}(x, y)$ for the 105 cases. The results are given in tables A-D section 5 and the methods used for this are mentioned in section 5 In section 1 we start with case a) of the Main Theorem as a typical example of the calculation of $H K R$ and its homological properties. In sections 2 and 3 we treat the cases c) and b) of out Main Theorem. Section 4 solves a question of Avramov and in section 5 we give the tables (tables A-D just mentioned) where hopefully all cases of $\Phi_{H K R}$ for the embedding dimension 4 cases (quadratic relations) have been calculated. We assume characteristic of $k$ to be 0 throughout except in section 6 where we present conjectures and some results for the higher embedding dimension cases.

We finally wish to thank Clas Löfwall, Jörgen Backelin and Victor Ufnarovski for stimulating discussions.

\section{A Koszul ring in 4 variables with 6 relations which is exceptional}

Recall first that if $H K R$ is the homology of the Koszul complex of a ring $R$ of embedding dimension $m$ as in the introduction, then there is a spectral sequence (the Avramov spectral sequence [AV1])

$$
E_{n}^{2}=\oplus_{p+q=n} \operatorname{Tor}_{p, q}^{H K R}(k, k)=>\operatorname{Tor}_{n}^{R}(k, k) / K R \otimes k
$$

leading to the inequality

$$
P_{R}(z) /(1+z)^{m} \leq \sum_{p \geq 0, q \geq 0, p+q=n}\left|\operatorname{Tor}_{p, q}^{H K R}(k, k)\right| z^{n}
$$

where we have equality if and only if the spectral sequence degenerates.

Let us now consider the ring

$$
R=k[x, y, z, u] /\left(x^{2}, x y, z u, x u, y^{2}+z^{2}, x z+u^{2}\right)
$$

- This is the case a) of the main theorem above. The Koszul dual of this ring is $k<X, Y, Z, U>$ $/(Y U-U Y, Y Z-Z Y, Y Y-Z Z, X Z-Z X-U U)$ and the Gröbner basis of this ideal is quadratic if we use the ordering of the variables $Y, U, Z, X$. Thus $R$ is a Koszul ring and its Hilbert series is $\left(1+3 t-2 t^{3}\right) /(1-t)$. However, the homology of the Koszul complex of $R$, namely $H K R$ and its homology are rather complicated: We now use the Macaulay2 package DGAlgebras with the input file:

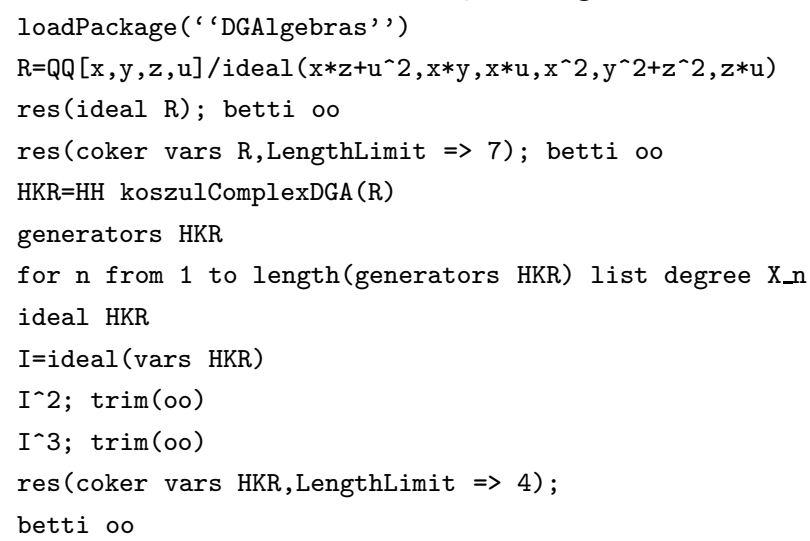

From the output file of this we get first the important fact that the algebra $H K R$ has the cube of its augmentation ideal $I=\overline{H K R}$ equal to 0 . This first result is useful for determining the homological properties of $H K R$, in particular for determining the series

$$
\Phi_{R}(x, y)=\sum_{p, q \geq 0}\left|\operatorname{Tor}_{p, q}^{H K R}(k, k)\right| x^{p} y^{q}
$$


Indeed, we can now use a general result by Clas Löfwall, which essentially says that many homological properties of a graded algebra, whose augmentation ideal has cube 0 are determined by its Hilbert series and the subalgebra of the whole Ext-algebra generated (under Yoneda product) of the $\operatorname{Ext}^{1}(k, k)$. More precisely, the theorem 1.3 on page 310 of [L1] (note that the citation page 19 in this Theorem should be page 309 loc.cit.). This theorem says that in our special case with the notations of loc. cit. our $\Phi_{R}(x, y)$ of (1) is equal to the Löfwall $\left.P_{H K R}(x, 1, y, 1)\right)$ and the Löfwall formula gives

$$
\Phi_{R}(x, y)=x H_{A}(x, y) /\left(1+x-H_{A}(x, y)\left(1-H_{I / I^{2}}(y) x+H_{I^{2}}(y) x^{2}\right)\right)
$$

where $H_{A}(x, y)$ is the Hilbert series in two variables of the algebra $A$ which is the subalgebra generated by $\operatorname{Ext}_{H K R}^{1}(k, k)$ of the Yoneda Ext-algebra $\operatorname{Ext}_{H K R}^{*}(k, k)$. This algebra is equal to the Koszul dual of $H K R$, which can be presented as

$$
T\left(\left(I / I^{2}\right)^{*}\right) /\left(\left(i m\left(I / I^{2} \otimes I / I^{2} \rightarrow I^{2}\right)^{*}\right)\right.
$$

where $T$ is the tensor algebra on $\left(I / I^{2}\right)^{*}$ which is the k-vector space dual of $I / I^{2}$ and $\left(i m\left(I / I^{2} \otimes I / I^{2} \rightarrow I^{2}\right)^{*}\right)$ is the twosided ideal in $T$ generated by the dual of the image of the natural multiplication map of $H K R$. It is thus natural to write $H K R^{!}$instead of $A$ and we will often write the preceding formula as

$$
1 / \Phi_{R}(x, y)=(1+1 / x) / H K R^{!}(x, y)-H K R(-x, y) / x
$$

where $H K R(x, y)$ is the Hilbert series in two variables of $H K R$. We now use this result and continue the analysis of the output file. The formula (3) shows that to get $\Phi_{R}(x, y)$ we need the Hilbert series $A(x, y)=H K R^{!}(x, y)$ of $A$ and the two Hilbert series $H_{I / I^{2}}(y)$ and $H_{I^{2}}(y)$, the most difficult part being the determination of $A(x, y)$ and we start with that. We see that that we can determine a presentation of $H K R$ in our special (and typical) case as having 6 generators $X_{1}, \ldots, X_{6}$ of degree $(1,2), 6$ generators $X_{7}, \ldots, X_{12}$ of degree $(2,3)$ and one generator $X_{13}$ of degree $(3,4)$ (here the second element of these pairs of degrees comes from the grading of $R$ and will be ignored), satisfying the following quadratic relations:

$$
\begin{gathered}
X_{5} X_{6}, X_{4} X_{6}, X_{2} X_{6}, X_{1} X_{6}, X_{4} X_{5}-X_{3} X_{6}, X_{2} X_{5}, X_{1} X_{5}, X_{2} X_{4}, X_{1} X_{4}, X_{1} X_{3}, X_{1} X_{2}, \\
X_{6} X_{12}, X_{5} X_{12}, X_{2} X_{12}, X_{1} X_{12}, X_{6} X_{11}-X_{4} X_{12}, X_{5} X_{11}-X_{3} X_{12}, X_{1} X_{11}, X_{6} X_{10}, \\
X_{5} X_{10}, X_{4} X_{10}, X_{2} X_{10}, X_{1} X_{10}, X_{6} X_{9}, X_{5} X_{9}, X_{4} X_{9}, X_{3} X_{9}+X_{4} X_{12}, X_{2} X_{9}, \\
X_{1} X_{9}, X_{6} X_{8}, X_{5} X_{8}-X_{4} X_{12}, X_{4} X_{8}, X_{3} X_{8}+X_{4} X_{11}, X_{2} X_{8}, X_{1} X_{8}, X_{6} X_{7}, X_{5} X_{7}, \\
X_{4} X_{7}, X_{3} X_{7}+X_{2} X_{11}, X_{2} X_{7}, X_{1} X_{7}, X_{6} X_{13}, X_{5} X_{13}, X_{4} X_{13}, X_{2} X_{13}, X_{1} X_{13}, X_{12} X_{12}, \\
X_{11} X_{12}, X_{10} X_{12}, X_{9} X_{12}, X_{8} X_{12}, X_{7} X_{12}, X_{10} X_{11}+X_{3} X_{13}, X_{9} X_{11}, X_{8} X_{11}, X_{7} X_{11}, X_{10} X_{10}, \\
X_{9} X_{10}, X_{8} X_{10}, X_{7} X_{10}, X_{9} X_{9}, X_{8} X_{9}, X_{7} X_{9}, X_{8} X_{8}, X_{7} X_{8}, X_{7} X_{7}, X_{12} X_{13}, X_{11} X_{13}, \\
X_{10} X_{13}, X_{9} X_{13}, X_{8} X_{13}, X_{7} X_{13}
\end{gathered}
$$

Now, using these relations we get that the Koszul dual $A$ of $H K R$ is the quotient of the free associative algebra $k<Y_{1}, \ldots, Y_{13}>$ on the dual generators $Y_{i}$ of the $X_{i}$ with the ideal generated by

$$
\begin{gathered}
{\left[Y_{2}, Y_{3}\right],\left[Y_{3}, Y_{4}\right],\left[Y_{3}, Y_{5}\right],\left[Y_{4}, Y_{5}\right]+\left[Y_{3}, Y_{6}\right],\left[Y_{3}, Y_{10}\right],\left[Y_{3}, Y_{11}\right], Y_{11} Y_{11},\left[Y_{3}, Y_{7}\right]-\left[Y_{2}, Y_{11}\right]} \\
{\left[Y_{5}, Y_{11}\right]+\left[Y_{3}, Y_{12}\right],\left[Y_{10}, Y_{11}\right]-\left[Y_{3}, Y_{13}\right],\left[Y_{3}, Y_{8}\right]-\left[Y_{4}, Y_{11}\right]} \\
{\left[Y_{4}, Y_{12}\right]+\left[Y_{6}, Y_{11}\right]+\left[Y_{5}, Y_{8}\right]-\left[Y_{3}, Y_{9}\right]}
\end{gathered}
$$

Here we have written e.g. $\left[Y_{2}, Y_{3}\right]=Y_{2} Y_{3}-Y_{3} Y_{2}$ and e.g. $\left[Y_{3}, Y_{13}\right]=Y_{3} Y_{13}-Y_{13} Y_{3}$ for the "odd" generators $Y_{i}, i=1 \ldots 6$ and $Y_{13}$. Furthermore we have written e.g. [ $\left.Y_{10}, Y_{11}\right]=Y_{10} Y_{11}+Y_{10} Y_{11}$ for the "even" generators and e.g. $\left[Y_{3}, Y_{10}\right]=Y_{3} Y_{10}+Y_{10} Y_{3}$ when the even and odd generators are mixed. Now to use the formula of Löfwall, we need to calculate the Hilbert series $A(x, y)$ of $A$ with BERGMAN [BA] using the presentation we have just detemined, where the bigrading of the preceding generators should now be 1 for the first degree (corresponding to $\mathrm{x}$ ) and for the second degree (corresponding to y) we should have 1 for $Y_{i}(i=1, \ldots 6)$, 
2 for $Y_{i}(i=7, \ldots 12)$ and 3 for $Y_{13}$. But BERGMAN can for the moment only directly handle Hilbert series of algebras where the generators have only degree 1 . But if for the moment we are only interested in the generating series of the total terms

$$
\Phi_{R}(x, x)=\sum_{n=0}^{\infty} \sum_{p+q=n}\left|\operatorname{Tor}_{p, q}^{H K R}(k, k)\right| x^{n}
$$

which occur in the Avramov spectral sequence it is sufficient to determine the Hilbert series $H_{A}(x, x)$ of A, i.e. to give the variables $Y_{i}(i=1, \ldots 6)$ the degree 2 , the variables $Y_{i}(i=7, \ldots 12)$ the degree 3 and the variable $Y_{13}$ the degree 4 , keep the relations as above and use the following Lemma that Victor Ufnarovski has been kind to communicate to me:

LEMMA (Ufnarovski) Let $A$ be a graded algebra which is quotient of a free algebra with $n$ generators $Y_{i}$ of degrees $d_{i} \geq 1$ by an ideal $I=\left(f_{1}, \ldots, f_{m}\right)$ which is homogeneous with respect to the given grading. Let now $B$ be a new graded algebra obtained from $A$ as follows: Introduce a new variable $t$ of degree 1 and new variables $Z_{i}$ of degrees 1 , and let $B$ be the quotient of the free associative algebra on the variables $t, Z_{i}$ with the "same" ideal as in $A$, but where we have replaced each $Y_{i}$ by $t^{d_{i}-1} Z_{i}$. Then we have the following relation between the Hilbert series of $A$ and $B$ :

$$
1 / A(z)=1 / B(z)+(n+1) z-\sum_{i=1}^{n} z^{d_{i}}
$$

i.e. we can reduce the calculation of $A(z)$ to the calculation of $B(z)$ which can be done directly in BERGMAN since all generators of $B$ have degree 1 .

The proof of the Lemma will be given at the end of this section.

Remark.There is an analogous result in Ufnarovski's book [U], Lemma on page 141.

We now continue with the proof of case a) of our main theorem:

We follow the recipe of the Ufnarovski Lemma. Recall that the variables $Y_{i}$ of degree $(1,2)$ should now be considered to have degree 2 etc.

We introduce a new variable $t$ of degree 1 and replace the variables $Y_{i}$ above as follows:

$$
Y_{i}=t Z_{i}, 1 \leq i \leq 6 ; Y_{i}=t^{2} Z_{i}, 7 \leq i \leq 12, Y_{13}=t^{3} Z_{13}
$$

where the new $Z_{i}$ :s are given the degree 1 . Now the algebra $A$ becomes the quotient of the free associative algebra in 14 generators of degree $1: k<Z_{1}, \ldots, Z_{6}, t, Z_{7} \ldots, Z_{13}>$ with the ideal above where e.g. $\left[Y_{2}, Y_{3}\right]$ is replaced by $t Z_{2} t Z_{3}-t Z_{3} t Z_{2}$ and $\left[Y_{10}, Y_{11}\right]-\left[Y_{3}, Y_{13}\right]$ is replaced by

$$
t^{2} Z_{10} t^{2} Z_{11}+t^{2} Z_{11} t^{2} Z_{10}-t Z_{3} t^{3} Z_{13}+t^{3} Z_{13} t Z_{3}
$$

etc. The new algebra is denoted by $B$ and the old one by $A$ and the Ufnarovski Lemma gives that we following relation between their Hilbert series $H_{A}(z)$ and $H_{B}(z)$ :

$$
1 / H_{A}(z)=1 / H_{B}(z)+14 z-6 z^{2}-6 z^{3}-z^{4}
$$

It remains to calculate $H_{B}(z)$. For this we can now directly use BERGMAN on the following input file which we call inbcaseB (we have written xi instead of $Z_{i}$ ):

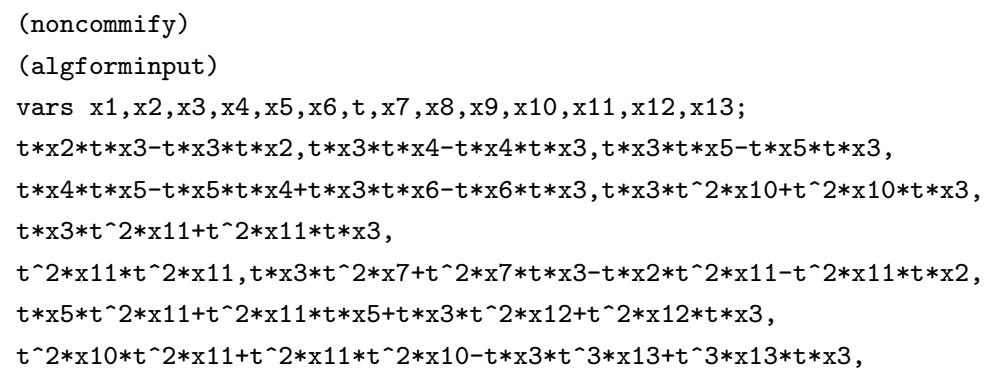




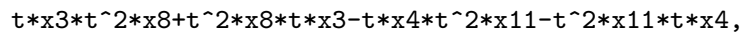

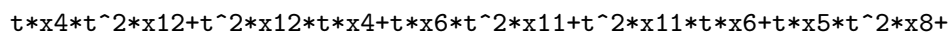

$\mathrm{t} \sim 2 * x 8 * t * x 5-t * x 3 * t \wedge 2 * x 9-t^{\wedge} 2 * x 9 * t * x 3$

The following command in BERGMAN:

(ncpbhgroebner ' 'inbcaseB', ' $t 1$ ', ' ' $t 2$ ', ' ' $t 3$ ')'

gives after a few seconds $t 3$, i.e. the Hilbert series for $B$ in degrees $\leq 20$ and $\geq 2$, so that

$$
1 / H_{B}(z)=1-14 z+4 z^{4}+6 z^{5}+2 z^{6}-z^{8}-z^{9}+z^{11}+z^{12}-z^{14}-z^{15}+z^{17}+z^{18}-z^{20}+O\left(z^{21}\right)
$$

leading to the guess (alternatively using the maple command convert ( " , ratpoly) on the previous formula (6)) that

$$
1 / H_{B}(z)=\frac{1-15 z+15 z^{2}-14 z^{3}+4 z^{4}+2 z^{5}+4 z^{7}+z^{8}}{1-z+z^{2}}
$$

But this is not a proof. To get a proof we will analyze the file t1, i.e. the groebner basis of the ideal in B. For the ordering of the variables we have given in inbcaseB this groebner basis is infinite. But, at my request some time ago Jörgen Backelin wrote an addition permutebreak.sl to the programme BERGMAN which checks the groebner bases up to a certain given degree of the different permutations of the variables of a given input to the programme BERGMAN. In the present case this seems rather discouraging in view of the fact that $14 !=87178291200$ but rather immediately we see that switching the variables $\mathrm{x} 2, \mathrm{x} 3$ to $\mathrm{x} 3, \mathrm{x} 2$ in the vars line of inbcaseB gives a finite groebner bases (in degrees $\leq 8$. Even better: after a short time with breaking degree 12, checking about 2700 cases, we obtain that the ordering $\mathrm{x} 1, \mathrm{x} 2, \mathrm{x} 4, \mathrm{x} 5, \mathrm{x} 6, \mathrm{t}, \mathrm{x} 3, \mathrm{x} 7, \mathrm{x} 8, \mathrm{x} 9, \mathrm{x} 10, \mathrm{x} 11, \mathrm{x} 12, \mathrm{x} 13$ gives a finite groebner bases with 4 elements of degree 2, 6 elements of degree 3 and 2 elements of degree 4 . We therefore get 12 leading monomials in the free algebra and the general structure of the Hilbert series follows from Govorov ([GOV],Theorem 2) and the formula (7) follows. Now the formula (5) gives that

$$
\begin{gathered}
1 / A(z)=\frac{1-15 z+15 z^{2}-14 z^{3}+4 z^{4}+2 z^{5}+4 z^{7}+z^{8}}{1-z+z^{2}}+14 z-6 z^{2}-6 z^{3}-z^{4}= \\
=\frac{1-z-5 z^{2}+3 z^{4}-3 z^{5}-z^{6}+4 z^{7}+z^{8}}{1-z+z^{2}}
\end{gathered}
$$

and now to use the Löfwall formula we only need to observe that $I^{2}$ is equal to

$$
X_{3} X_{6}, X_{3} X_{5}, X_{3} X_{4}, X_{2} X_{3}, X_{4} X_{12}, X_{3} X_{12}, X_{4} X_{11}, X_{3} X_{11}, X_{2} X_{11}, X_{3} X_{10}, X_{3} X_{13}, X_{11} X_{11}
$$

and $I^{3}=0$ so that

$$
H K R(x, y)=1+6 x y+6 x y^{2}+x y^{3}+4 x^{2} y^{2}+6 x^{2} y^{3}+2 x^{2} y^{4}
$$

and

$$
\begin{aligned}
1 / \Phi_{R}(z, z) & =(1+1 / z) / A(z)-\left(1-6 z^{2}-6 z^{3}+3 z^{4}+6 z^{5}+2 z^{6}\right) / z= \\
& =\frac{(1+z)\left(1-2 z-3 z^{2}+3 z^{3}-3 z^{5}+2 z^{6}+z^{7}\right)}{1-z+z^{2}}
\end{aligned}
$$

Now, since $R$ is a Koszul ring with Hilbert series $P_{R}(z)=\frac{1+z}{1-3 z+2 z^{3}}$ given above, it follows in particular that if the Avramov spectral sequence degenerated, we would have had $P_{R}(z) /(1+z)^{4}=\Phi_{R}(z)$. But

$$
(1+z)^{4} / P_{R}(z)-1 / \Phi_{R}(z)=\frac{z^{7}(1+z)}{1-z+z^{2}}
$$

shows that this is not the case (there is a non-zero Massey product in $K R$ of degree 7 ). 
Remark: In the last section we will see how to calculate the series of $\Phi_{R}(x, y)$ using the extra degree in $H K R$ coming from the Koszul homology. There is also a third degree, coming from the grading of $R$, that we will not use.

PROOF OF THE UFNAROVSKI LEMMA (UFNAROVSKI): We need the theory of $n$-chains [U] of an algebra such as $A=k<Y_{1}, Y_{2}, \ldots>/\left(f_{1}, \ldots f_{m}\right)$. We use deglex order. It is known (cf. [U],p.51) that

$$
1 / A(z)=1-H_{X}(z)+H_{C_{1}}(z)-H_{C_{2}}(z)+H_{C_{3}}(z)-\ldots
$$

where $X=Y_{1}, Y_{2}, \ldots$ are the generators of $A$, and $H_{X}(z)=\sum z^{d_{i}}$ is the Hilbert series (i.e. the generating series) of $\mathrm{X}$, and where $C_{1}=$ the leading terms of the relations $f_{i}$ (we use the deglex order) and $H_{C_{1}}(z)$ is the generating series of $C_{1}$, similarly for the $n$-chains $C_{n}$ for $n \geq 2$. We now introduce an auxiliary algebra $D$ which I write

$$
D=<Y_{1}, Y_{2}, \ldots, t, Z_{1}, Z_{2}, \ldots>/\left(f_{1}, \ldots, f_{m}, Y_{i}-t^{d_{i}-1} Z_{i} \ldots>\right.
$$

Now if the order is deglex such that

$$
Y_{1}>Y_{2}>\ldots>t>Z_{1}>Z_{2}>\ldots Z_{n}
$$

For this algebra $D$ we have the new $X^{\prime}=X \bigcup\left\{t, Z_{1}, \ldots, Z_{n}\right\}$ and the new

$$
C_{1}^{\prime}=C_{1} \bigcup\left\{t^{d_{i}-1} Z_{i}\right\}
$$

but the higher $C_{i}$ for $i \geq 2$ are the same. It follows that

$$
H_{D}(z)=H_{A}(z)-(n+1) z+\sum_{i=1}^{n} z^{d_{i}}
$$

On the other hand, consider the order

$$
Y_{1}>Y_{2}>\ldots Z_{1}>Z_{2}>\ldots>t
$$

We have that $Y_{i}$ can be replaced by $t^{d_{i}-1} Z_{i}$ and we get the algebra

$$
B=k<Z_{1}, Z_{2}, \ldots Z_{n}, t>/\left(g_{1}, g_{2}, \ldots, g_{m}\right)
$$

where the relations $g_{i}$ are obtained from the $f_{i}$ by replacing all $Y_{i}$ by $t^{d_{i}-1} Z_{i}$ Clearly $H_{B}(z)=H_{D}(z)$ and the lemma is proved.

\section{One more exceptional case.}

This case will be treated as in section 1 so we can be more brief here. The is the case $c)$ in the main Theorem of section 0 i.e. the ring $R_{60 v a}=k[x, y, z, u] /\left(x^{2}+y z+u^{2}, x z+y u, x u, x^{2}+x y, z^{2}, x u\right)$. The resolution of the ideal of $R_{60 v a}$ over $k[x, y, z, u]$ has betti numbers:

$$
\left(\begin{array}{ccccc}
1 & \cdot & \cdot & \cdot & \cdot \\
\cdot & 6 & 5 & \cdot & \cdot \\
\cdot & \cdot & 6 & 9 & 3
\end{array}\right)
$$

and an applications of the programme DGAlgebras gives again that the algebra $H K R_{60 v a}$ has the cube of the augmentation ideal $\overline{H K R_{60 v a}}$ equal to 0 . Furthermore we have 6 generators $X_{1}, \ldots, X_{6}$ of degree $(1,2)$ and 5 generators $X_{7}, \ldots, X_{11}$ of degree $(2,3)$ satisfying the following quadratic relations:

$$
\begin{gathered}
X_{5} X_{6}, X_{3} X_{6}+X_{4} X_{6}, X_{1} X_{6}, X_{4} X_{5}-X_{2} X_{6}, X_{3} X_{5}+X_{2} X_{6}, X_{2} X_{5}, X_{1} X_{5}, X_{1} X_{4}, X_{1} X_{2} \\
X_{6} X_{11}, X_{5} X_{11}, X_{3} X_{11}+X_{4} X_{11}, X_{1} X_{11}, X_{6} X_{10}, X_{5} X_{10}, X_{4} X_{10}-X_{2} X_{11}, X_{2} X_{10} \\
X_{1} X_{10}, X_{6} X_{9}-X_{4} X_{11}, X_{5} X_{9}-X_{2} X_{11}, X_{1} X_{9}, X_{6} X_{8}, X_{5} X_{8}, X_{3} X_{8}+X_{4} X_{8}
\end{gathered}
$$




$$
\begin{gathered}
X_{1} X_{8}+X_{3} X_{10}+X_{2} X_{11}, X_{6} X_{7}-X_{2} X_{11}, X_{5} X_{7}, X_{4} X_{7}+X_{2} X_{9}, X_{2} X_{7}, X_{1} X_{7}, X_{11} X_{11}, \\
X_{10} X_{11}, X_{9} X_{11}, X_{8} X_{11}, X_{7} X_{11}, X_{10} X_{10}, X_{9} X_{10}, X_{8} X_{10}, X_{7} X_{10}, X_{7} X_{9}, X_{8} X_{8}, X_{7} X_{7}
\end{gathered}
$$

Now, using these relations we get that the Koszul dual of $H K R$ is the quotient of the free algebra $k<$ $Y_{1}, \ldots, Y_{11}>$ on the dual generators $Y_{i}$ of the $X_{i}$ with the ideal generated by

$$
\begin{gathered}
{\left[Y_{1}, Y_{3}\right],\left[Y_{2}, Y_{3}\right],\left[Y_{3}, Y_{4}\right],\left[Y_{2}, Y_{4}\right],-\left[Y_{3}, Y_{6}\right]+\left[Y_{4}, Y_{6}\right],\left[Y_{4}, Y_{5}\right]+\left[Y_{2}, Y_{6}\right]-\left[Y_{3}, Y_{5}\right],\left[Y_{2}, Y_{8}\right]} \\
{\left[Y_{3}, Y_{7}\right],\left[Y_{3}, Y_{9}\right],\left[Y_{4}, Y_{9}\right],\left[Y_{7}, Y_{8}\right],\left[Y_{8}, Y_{9}\right], Y_{9} Y_{9},-\left[Y_{3}, Y_{11}\right]+\left[Y_{4}, Y_{11}\right]+\left[Y_{6}, Y_{9}\right],} \\
{\left[Y_{4}, Y_{10}\right]+\left[Y_{2}, Y_{11}\right]+\left[Y_{5}, Y_{9}\right]-\left[Y_{1}, Y_{8}\right]+\left[Y_{6}, Y_{7}\right],-\left[Y_{3}, Y_{8}\right]+\left[Y_{4}, Y_{8}\right],-\left[Y_{1}, Y_{8}\right]+\left[Y_{3}, Y_{10}\right],} \\
-\left[Y_{4}, Y_{7}\right]+\left[Y_{2}, Y_{9}\right]
\end{gathered}
$$

Here we have again written e.g. $\left[Y_{1}, Y_{3}\right]=Y_{1} Y_{3}-Y_{3} Y_{1}$ for the "odd" generators $Y_{i}, i=1 \ldots 6$, and e.g. $\left[Y_{8}, Y_{9}\right]=Y_{8} Y_{9}+Y_{9} Y_{8}$ for the "even" generators $Y_{i}, i=7 \ldots 11$, and e.g. $\left[Y_{2}, Y_{8}\right]=Y_{2} Y_{9}+Y_{8} Y_{2}$ when even and odd generators are mixed. We want to calculate the Hilbert series of this quotient, when the generators $Y_{i}$ are given the degree 2 for $i=1, \ldots, 6$ and degree 3 for $i=7, \ldots, 11$. For this we use again the programme BERGMAN and the idea of Victor Ufnarovski to handle the gradings, i.e. we introduce a new variable $t$ of degree 1 and replace the variables $Y_{i}$ in (9)

$$
Y_{i}=t Z_{i}, 1 \leq i \leq 6 ; Y_{i}=t^{2} Z_{i}, 7 \leq i \leq 11
$$

where the $Z_{i}$ :s also have degree 1 . Now the algebra $A$ becomes the quotient of the free algebra in 12 generators of degree 1: $k<Z_{1}, \ldots, Z_{6}, t, Z_{7}, \ldots, Z_{11}>$ with the ideal (9) above where e.g. $\left[Y_{1}, Y_{3}\right]$ is replaced by $t Z_{1} t Z_{3}-t Z_{3} t Z_{1}$ and e.g. $Y_{9} Y_{9}$ is replaced by $t^{2} Z_{9} t^{2} Z_{9}$ and $-\left[Y_{4}, Y_{7}\right]+\left[Y_{2}, Y_{9}\right]$ is replaced by

$$
-t Z_{4} t^{2} Z_{7}-t^{2} Z_{7} t Z_{4}+t Z_{2} t^{2} Y_{9}+t^{2} Y_{9} t Z^{2}
$$

etc. If the new algebra is denoted by $B$ and the old one by $A$, then by the theory of Ufnarovski we have the following relation between their Hilbert series:

$$
1 / H_{A}(z)=1 / H_{B}(z)+12 z-6 z^{2}-5 z^{3}
$$

It remains to calculate $H_{B}(z)$.

After these preparations we are now ready again to use the programme BERGMAN and the command in BERGMAN

(ncpbhgroebner "inbcaseB" " $\mathrm{t} 1^{\prime \prime} \quad$ " $\mathrm{t} 2$ " " $\mathrm{t} 3^{\prime \prime}$ )

After a few seconds we get $\mathrm{t} 3$ (i.e. the Hilbert series for $B$ ) in degrees $\leq 20$ and $\geq 2$ and we obtain with maple

$$
\begin{aligned}
S:= & \operatorname{series}\left(1 /(1+12 z+\mathrm{t} 3, z, 21)=1-12 z+6 z^{4}+9 z^{5}+2 z^{6}-3 z^{7}-4 z^{8}-2 z^{9}+z^{10}\right. \\
& +3 z^{11}+2 z^{12}-z^{13}-3 z^{14}-2 z^{15}+z^{16}+3 z^{17}+2 z^{18}-z^{19}-3 z^{20}+O\left(z^{21}\right)
\end{aligned}
$$

and the maple command convert ( $\mathrm{S}$, ratpoly) on the previous series gives the rational function:

$$
\frac{(1-z)\left(z^{9}+2 z^{8}+z^{7}-3 z^{6}-2 z^{5}-5 z^{4}-11 z^{3}+z^{2}-12 z+1\right)}{\left(1-z+z^{2}\right)}
$$

Here is again how one should proceed to get a proof of this formula. If we can find a permutation of the 12 variables in inbcaseB so that the groebner basis $t 1$ is finite, then the Hilbert series of $B$ is equal to the Hilbert series of the free algebra on the 12 variables divided by the ideal generated by the monomial leading terms of this finite groebner basis, and this last Hilbert series is rational of a special form according to Govorov. Again we use Jörgen Backelin's addition permutebreak.sl to the programme BERGMAN and we 
obtain that the order of the variables $x 3, t, x 1, x 2, x 5, x 6, x 4, y 8, y 7, y 9, y 10, y 11$ gives a finite groebner basis with 6 elements of degree 4,9 elements of degree 5 and 3 elements of degree 6 . We therefore get 18 leading monomials in the free algebra and the general structure of the Hilbert series follows from Govorov ([GOV], Theorem 2) and the formula (9) follows. We have now all we need to prove the following

THEOREM Let $R_{60 v a}=k[x, y, z, u] /\left(x^{2}+y z+u^{2}, x z+y u, z u, x^{2}+x y, z^{2}, x u\right)$. Then

i)

$$
\begin{gathered}
\Phi_{R 60 v a}(z, z)= \\
\sum_{n=0}^{\infty} \sum_{p+q=n}\left|\operatorname{Tor}_{p, q}^{H K R}(k, k)\right| z^{n}=\frac{\left(1-z+z^{2}\right)}{(1+z)\left(z^{9}-z^{8}+z^{7}+z^{6}-4 z^{5}+z^{4}+4 z^{3}-3 z^{2}-2 z+1\right)}
\end{gathered}
$$

ii) $P_{R}(z)=(1+z) /\left(-z^{6}-z^{5}+3 z^{3}-3 z+1\right)$

iii) $1 / \Phi_{R 60 v a}(z, z)-(1+z)^{4} / P_{R 60 v a}(z)=z^{7}(1+z)\left(1-3 z^{2}-z^{3}\right) /\left(1-z-z^{2}\right)$

It follows that the Avramov spectral sequence does not degenerate (the two series differ starting in degree 7).

PROOF: For the algebra $H K R$ we have that the cube of the augmentation ideal $\overline{H K R}$ is zero. Furthermore the rational function $1 / H K R^{!}(z)$ is given by the formula (9) above and the two-variable form of the Hilbert series of $H K R$ is given by the formula

$$
H K R(x, y)=1+6 x y+5 x y^{2}+6 x^{2} y^{2}+9 x^{2} y^{3}+3 x^{2} y^{4}
$$

This gives the one-variable Hilbert series $H(-z, z)$ and the formula

$$
1 / P_{H K R}(z)=(1+1 / z) / H K R^{!}(z)-H(-z, z) / z
$$

gives the result i).

To prove ii) we first observe that the $R^{!}=k<X, Y, Z, U>/\left(Y^{2}, X^{2}-X Y-Y X-Y Z-Z Y, X Z+\right.$ $\left.Z X-Y U-U Y, Y Z+Z Y-U^{2}\right)$, where the $X, Y, Z, U$ are the dual variables to $x, y, z, u$. There are only 24 permutations of the variables $X, Y, Z, U$ and one sees that the order $X, Y, U, Z$ gives in BERGMAN a finite Gröbner basis, and that $1 / R^{!}(z)=(1-z)^{2}\left(1-2 z-z^{2}-z^{3}\right)$. On the other hand the Hilbert series $R(z)=\left(1-3 z+3 z^{3}\right) /(1-z)=1+4 z+4 z^{2}+z^{3}+z^{4}+z^{5}+\ldots$ and according to Theorem B.9 by Clas Löfwall (cf. page 310 of [R3] the ring $R$ satisfies a condition $\mathcal{M}_{3}$ so that we still have the formula $1 / P_{R}(z)=(1+1 / z) / R^{!}(z)-R(-z) / z$ and this gives ii) and the assertion iii) follows.

\section{One final exceptional case for a local ring that is artinian and a Koszul algebra.}

In section 4 we will treat a Koszul local ring (case 46) whose Koszul algebra has properties that show that the Avramov spectral sequence degenerates (solving a problem of Avramov) In section 2 we found a quadratic local ring (case 60va) whose Avramov spectral sequence does not degenerate. But case 60va is not a Koszul algebra. Now we pass to a case of a Koszul local ring (case 71) which has further rather unexpected properties and which is case b) of the Main Theorem.

THEOREM.- There are at least five local commutative rings having Hilbert series $(1+z)(1+3 z)$ which are Koszul algebras and which have different homological properties. Furthermore for one of these five cases the Avramov spectral sequence does not degenerate.

PROOF: For the ring of case 71 in the Appendix 1 we have the presentation

$$
R_{71}=k[x, y, z, u] /\left(x^{2}, y^{2}, z^{2}, u^{2}, x y, z u, y z+x u\right)
$$

But we also have three other rings which are Koszul algebras and have the same Hilbert series:

$$
R_{71 v 4}=\frac{k[x, y, z, u]}{\left(x^{2}+u^{2}, x y, x u, y^{2}, y z, z^{2}, z u\right)} R_{71 v 7}=\frac{k[x, y, z, u]}{\left(x^{2}, y^{2}, z^{2}, x z+u^{2}, x u, y z, z u\right)} R_{71 v 5}=\frac{k[x, y, z, u]}{\left(x^{2}+x y, x^{2}+y z, x y+y^{2}, z^{2}, x u, z u, x z+u^{2}\right)}
$$


and but the betti numbers of the resolution of the preceding four ideals are:

$$
\left(\begin{array}{lllll}
1 & . & . & . & \cdot \\
\cdot & 7 & 8 & 2 & . \\
\cdot & \cdot & 5 & 8 & 3
\end{array}\right) \quad\left(\begin{array}{lllll}
1 & . & . & . & . \\
\cdot & 7 & 8 & 3 & . \\
. & . & 6 & 8 & 3
\end{array}\right) \quad\left(\begin{array}{lllll}
1 & . & . & . & . \\
\cdot & 7 & 8 & 1 & . \\
. & . & 4 & 8 & 3
\end{array}\right) \quad\left(\begin{array}{lllll}
1 & . & . & . & . \\
\cdot & 7 & 8 & . & . \\
. & . & 3 & 8 & 3
\end{array}\right)
$$

so that the rings can not be isomorphic. But their $H K R$ are rather easy, in one of the cases $H K R$ has only monomial relations, But strangely enough the case of a $R_{71}$ with only monomial relations gives a more complicated $H K R$.

But there remains one case $R_{71 v 16}$ which is very different from the preceding and leads to a new phenomenon. We now treat it in detail: The ring

$$
R_{71 v 16}=k[x, y, z, u] /\left(x z+u^{2}, x y, x u, x^{2}, y^{2}+z^{2}, z u, y z\right)
$$

is also a Koszul algebra and it has the same matrix of Betti numbers as $R_{71}$ but the algebraic behavior of its $H K R$ is very different. It still has the cube of the augmentation ideal of $H K R$ equal to 0 , it has seven $X_{1}, \ldots, X_{7}$ generators of degree $(1,2)$, eight generators $X_{8}, \ldots, X_{15}$ of degree $(2,3)$ and two generators $X_{16}, X_{17}$ of degree $(3,4)$ and an applications of the programme DGAlgebras gives that these generators satisfy 128 quadratic relations of which however only the following 16 are non-monomial relations:

$$
\begin{gathered}
X_{5} X_{6}-X_{3} X_{7}, X_{2} X_{3}-X_{4} X_{5}, X_{7} X_{13}-X_{5} X_{15}, X_{6} X_{13}-X_{3} X_{15}, X_{3} X_{12}-X_{5} X_{14} \\
X_{3} X_{11}+X_{5} X_{15}, X_{6} X_{10}-X_{5} X_{15}, X_{4} X_{10}-X_{2} X_{13}, X_{3} X_{10}+X_{5} X_{13}, X_{5} X_{9}+X_{2} X_{13} \\
X_{3} X_{9}+X_{4} X_{13}, X_{3} X_{8}+X_{2} X_{13}, X_{3} X_{16}-X_{5} X_{17}, X_{13} X_{14}+X_{3} X_{17}, X_{10} X_{14}+X_{5} X_{17} \\
X_{12} X_{13}+X_{5} X_{17}
\end{gathered}
$$

and since the following 6 quadratic monomials do not occur among the 128 relations:

$$
X_{3} X_{4}, X_{3} X_{5}, X_{3} X_{6}, X_{3} X_{13}, X_{3} X_{14}, X_{13} X_{13}
$$

it follows that the Koszul dual $H K R^{!}$is equal to the quotient of the free associative algebra on the 17 variables variables $Y_{i}, 1 \leq i \leq 17$ (which are dual to the $X_{i}$ ) with respect to the ideal generated by the 16 quadratic relations:

$$
\begin{gathered}
{\left[Y_{3}, Y_{4}\right],\left[Y_{3}, Y_{5}\right],\left[Y_{3}, Y_{6}\right],\left[Y_{3}, Y_{13}\right],\left[Y_{3}, Y_{14}\right], Y_{13} Y_{13},\left[Y_{2}, Y_{3}\right]+\left[Y_{4}, Y_{5}\right],\left[Y_{5}, Y_{6}\right]+\left[Y_{3}, Y_{7}\right]} \\
{\left[Y_{3}, Y_{12}\right]+\left[Y_{5}, Y_{14}\right],\left[Y_{3}, Y_{10}\right]-\left[Y_{5}, Y_{13}\right],\left[Y_{3}, Y_{15}\right]+\left[Y_{6}, Y_{13}\right],\left[Y_{3}, Y_{9}\right]-\left[Y_{4}, Y_{13}\right],} \\
{\left[Y_{3}, Y_{17}\right]-\left[Y_{13}, Y_{14}\right],\left[Y_{2}, Y_{13}\right]-\left[Y_{3}, Y_{8}\right]+\left[Y_{4}, Y_{10}\right],\left[Y_{5}, Y_{15}\right]+\left[Y_{7}, Y_{13}\right]-\left[Y_{6}, Y_{10}\right]-\left[Y_{3}, Y_{11}\right],} \\
{\left[Y_{5}, Y_{17}\right]-\left[Y_{12}, Y_{13}\right]-\left[Y_{10}, Y_{14}\right]+\left[Y_{3}, Y_{16}\right]}
\end{gathered}
$$

Here [,] means as before the graded commutator so that $\left[Y_{i}, Y_{j}\right]=Y_{i} Y_{j}-Y_{j} Y_{i}$ if $1 \leq i \leq 7$ or $i=16$ or $i=17$ and $1 \leq j \leq 7$ or $j=16$ or $j=17$. Furthermore we have $\left[Y_{s}, Y_{t}\right]=Y_{s} Y_{t}+Y_{t} Y_{s}$ otherwise. We now need to calculate the Hilbert series of $H K R^{!}$where we have given the variables $Y_{1}, \ldots, Y_{7}$ the degrees 2, the variables $Y_{8}, \ldots, Y_{15}$ the degrees 3 and the variables $Y_{16}, Y_{17}$ the degrees 4 . We use the Ufnarovski trick and introduce a new variable $T$ of degree 1 and replace everywhere in $H K R^{!}$the variables $Y_{i}$ according to the formulae $Y_{i}=T Z_{i}$ for $1 \leq i \leq 7, Y_{i}=T^{2} Z_{i}$ for $8 \leq i \leq 15$ and $Y_{i}=T^{3} Z_{i}$ for $\mathrm{i}=16,17$. The modified algebra $A$ has generators of degree 1 and can be put into BERGMAN and its Hilbert series $A(z)$ is related to $H K R^{!}(z)$ by the Ufnarovski formula:

$$
\frac{1}{H K R^{!}(z)}=\frac{1}{A(z)}+18 z-7 z^{2}-8 z^{3}-2 z^{4}
$$

The calculations in BERGMAN gives after a few minutes $A(z)$ up to degree 22, giving that

$$
1 / A(z)=1-18 z+5 z^{4}+8 z^{5}+3 z^{6}-z^{8}-z^{8}+z^{11}+z^{12}-z^{14}-z^{15}+z^{17}+z^{18}-z^{20}-z^{21}+O\left(z^{23}\right)
$$


indicating (using the maple command convert(-,ratpoly) as earlier) that

$$
\frac{1}{A(z)}=\frac{1-19 z+19 z^{2}-18 z^{3}+5 z^{4}+3 z^{5}+5 z^{7}+2 z^{8}}{1-z+z^{2}}
$$

To prove this we use again the permutebergman addition by Jörgen Backelin to BERGMAN and we try to find a permutation of the variables $T, Z_{i}, i=1 \ldots 17$ that gives a finite Gröbner basis when we calculate the Hilbert series of the algebra $A$. Since the number of permutations of 18 variables is $18 !=6402373705728000$ this seems to be a hopeless task, but after letting the programme run through only the 24000 first permutations (this took about an hour) we finally got the permutation

$$
Z 1, Z 3, Z 4, Z 6, Z 7, T, Z 2, Z 5, Z 8, Z 9, Z 10, Z 11, Z 12, Z 13, Z 14, Z 15, Z 16, Z 17
$$

for which the Gröbner basis is finite: it has 5 elements of degree 4, 8 elements of degree 5 and 3 elements of degree 6. Therefore, according to the result of Govorov the Hilbert series $A(z)$ is rational as indicated. We have now all we need to prove the following

THEOREM Let $R=k[x, y, z, u] /\left(x z+u^{2}, x y, x u, x^{2}, y^{2}+z^{2}, z u, y z\right)$. Then $R$ is a Koszul algebra with Hilbert series $R(z)=1+4 z+3 z^{2}$ so that $P_{R}(z)=1 /\left(1-4 z+3 z^{2}\right)$. But $H K R$ is far from being a Koszul algebra. Indeed:

a)

$$
\begin{gathered}
\Phi_{R}(z, z)=\sum_{n=0}^{\infty} \sum_{p+q=n}\left|\operatorname{Tor}_{p, q}^{H K R}(k, k)\right| z^{n}= \\
\frac{\left(1-z+z^{2}\right)}{(1+z)\left(2 z^{7}+2 z^{6}-4 z^{5}+z^{4}+3 z^{3}-4 z^{2}-2 z+1\right)}
\end{gathered}
$$

b) Furthermore $P_{R}(z)=1 /\left(1-4 z+3 z^{2}\right)$ so that

$$
(1+z)^{4} / P_{R}(z)-1 / \Phi_{R}(z, z)=z^{7}(1+z) /\left(1-z+z^{2}\right)
$$

It follows again that the Avramov spectral sequence does not degenerate.

PROOF: We have just determined $1 / A(z)$ and it follows that

$$
\frac{1}{H K R^{!}(z, z)}=\frac{1-z-6 z^{2}-z^{3}+4 z^{4}-3 z^{5}-2 z^{6}+5 z^{7}+2 z^{8}}{1-z+z^{2}}
$$

But the two-variable version of the Hilbert series of $H K R$ is

$$
H K R(x, y)=1+7 x y+8 x y^{2}+2 x * y^{3}+5 x^{2} y^{2}+8 x^{2} y^{3}+3 x^{2} y^{4}
$$

This gives again a one-variable $H K R(-z, z)=1-7 z^{2}-8 z^{3}+3 z^{4}+8 z^{5}+3 z^{6}$ The formula by Clas Löfwall $1 / P_{H K R}(z, z)=(1+1 / z) / H K R^{!}(z, z)-H K R(-z, z) / z$ now gives

$$
1 / P_{H K R}(z, z)=\frac{(1+z)\left(1-2 z-4 z^{2}+3 z^{3}+z^{4}-4 z^{5}+2 z^{6}+2 z^{7}\right)}{1-z+z^{2}}
$$

and the Theorem is proved.

\section{The other embedding dimension four cases.}

We have just treated the three interesting cases of the Main Theorem in section 0. For the other cases we have that the Avramov spectral sequence does degenerate and they are treated in an analogous way. Here we will only treat case 46 in detail because it solves a problem that was left open in Avramov [AV1]. The case 46 in the ring $k[x, y, z, u] /\left(x^{2}, x y, y z, z u, u^{2}\right)$. 
Application as before of the programme DGAlgebras gives that the algebra $H K R$ has the cube of the augmentation ideal equal to 0 and that $H K R$ has 5 generators $X_{1}, \ldots, X_{5}$ of degree $(1,2)$ and 4 generators $X_{6}, X_{7}, X_{8}, X_{9}$ of degree $(2,3)$. These generators have only quadratic relations and they are:

$$
\begin{gathered}
X_{4} X_{5}, X_{3} X_{5}, X_{2} X_{5}, X_{3} X_{4}, X_{2} X_{3}, X_{1} X_{3}, X_{1} X_{2}, X_{5} X_{9}, X_{4} X_{9}, X_{3} X_{9}, X_{2} X_{9}, \\
X_{5} X_{8}, X_{4} X_{8}, X_{3} X_{8}, X_{5} X_{7}, X_{4} X_{7}-X_{2} X_{8}, X_{3} X_{7}, X_{2} X_{7}, X_{1} X_{7}, X_{5} X_{6}-X_{1} X_{9}, \\
X_{3} X_{6}, X_{2} X_{6}, X_{1} X_{6}, X_{9} X_{9}, X_{8} X_{9}, X_{7} X_{9}, X_{6} X_{9}, X_{8} X_{8}, X_{7} X_{8}, X_{7} X_{7}, X_{6} X_{7}, X_{6} X_{6}
\end{gathered}
$$

so that the Koszul dual $H K R^{!}$is the quotient of the free algebra $k<Y_{1}, \ldots, Y_{9}>$ in the dual generators $Y_{i}$ of the $X_{i}$ with the ideal generated by

$$
\left[Y_{1}, Y_{4}\right],\left[Y_{1}, Y_{5}\right],\left[Y_{2}, Y_{4}\right],\left[Y_{1}, Y_{8}\right],\left[Y_{4}, Y_{6}\right],\left[Y_{6}, Y_{8}\right],\left[Y_{4}, Y_{7}\right]+\left[Y_{2}, Y_{8}\right],\left[Y_{5}, Y_{6}\right]+\left[Y_{1}, Y_{9}\right]
$$

Here again we have written e.g. $\left[Y_{1}, Y_{4}\right]=Y_{1} Y_{4}-Y_{4} Y_{1}$ for the "odd" generators and e.g. $\left[Y_{6}, Y_{8}\right]=Y_{2} Y_{8}+Y_{8} Y_{2}$ for the "even" generators and e.g. $\left[Y_{4}, Y_{7}\right]=Y_{4} Y_{7}+Y_{7} Y_{4}$ when the even and odd generators are mixed. We use the previous methods to calculate the Hilbert series of our quotient when the generators $Y_{1}, \ldots, Y_{5}$ are given the degrees 2 and the generators $Y_{6}, \ldots, Y_{9}$ are given the degree 3 , i.e. we introduce a new variable $t$ of degree 1 and replace the variables $Y_{i}$ by $t Z_{i}$ for $1 \leq i \leq 5$ and $t^{2} Z_{i}$ for $6 \leq i \leq 9$. where the $Z_{i}$ now have degree 1. Our algebra $H K R$ ! is now replaced by the quotient of $k<Z_{1}, \ldots, Z_{5}, t, Z_{6}, \ldots, Z_{9}>$ by the ideal of $H K R^{!}$where e.g. $Y_{1} Y_{4}-Y_{4} Y_{1}$ is replaced by $t Z_{1} t Z_{4}-t Z_{4} t Z_{1}$ etc. If this new algebra is denoted by $B$ then again by the theory of Ufnarovskij we have in this special case the following relation between the their Hilbert series $H_{A}(z)$ and $H_{B}(z)$

$$
1 / H_{A}(z)=1 / H_{B}(z)+10 z-5 z^{2}-4 z^{3}
$$

and $H_{B}(z)$ is calculated by BERGMAN as before. We get:

$$
1 / H_{B}(z)=1-10 z+3 z^{4}+4 z^{5}+z^{6}
$$

since the permutation $Z_{1}, Z_{2}, Z_{3}, t, Z_{4}, \ldots, Z_{9}$ of the variables in $B$ gives a finite groebner basis (3 elements of degree 4,4 elements of degree 5 and one element of degree 6 ).

We are now ready to calculate $\Phi(x, x)$ of our $H K R$. We have the formula of Löfwall

$$
1 / \Phi_{R}(z)=(1+1 / z) / A(z)-H K R(-z, z) / z
$$

where we have just found that

$$
1 / A(z)=\left(1-10 z+3 z^{4}+4 z^{5}+z^{6}\right)+10 z-5 z^{2}-4 z^{3}=1-5 z^{2}-4 z^{3}+3 z^{4}+4 z^{5}+z^{6}
$$

For the Hilbert series in two variables of $H K R$ we observe that since

$$
\overline{H K R}^{2}=X_{1} X_{5}, X_{2} X_{4}, X_{1} X_{4}, X_{1} X_{9}, X_{2} X_{8}, X_{1} X_{8}, X_{4} X_{6}, X_{6} X_{8}
$$

that

$$
H K R(x, y)=1+5 x y+4 x y^{2}+3 x^{2} y^{2}+4 x^{2} y^{3}+x^{2} y^{4}
$$

so that $\operatorname{HKR}(-z, z)=1-5 z^{2}-4 z^{3}+3 z^{4}+4 z^{5}+z^{6}$ so that $1 / \Phi_{R}(z)=1-5 z^{2}-4 z^{3}+3 z^{4}+4 z^{5}+z^{6}$ too. But since $R$ is a Koszul ring (quadratic monomial relations) with Hilbert series $H_{R}(z)=\left(1+3 z+z^{2}-z^{3}\right) /(1-z)$ we have that (Fröberg) $P_{R}(z)=1 / H_{R}(-z)$ we have $(1+z)^{4} / P_{R}(z)=1-5 z^{2}-4 z^{3}+3 z^{4}+4 z^{5}+z^{6}$ too and therefore the Avramov spectral sequence degenerates.

\section{More precise $\Phi_{R}(x, y)$}

In the preceding sections we have shown how to calculate $\Phi_{R}(x, x)$ for some examples $R$. In this section we will given an indication about how to generalize this to the calculation of the double series $\Phi_{R}(x, y)$. The complete calculation results for the quadratic embedding dimension $\leq 4$ case are given in Tables A-D 
below. Let us illustrate these more precise calculations with the simple example of case 46 which we have just treated for the calculation of $\Phi_{R}(x, x)$. Note first that the output file for the case 46 gives for the dimensions of the $\operatorname{Tor}_{p, q}^{H K R}(k, k)$ the integers:

\begin{tabular}{|c|c|c|c|c|c|c|c|c|}
\hline 0: & 1 & 5 & 22 & 95 & 409 & 1760 & 7573 & 32585 \\
\hline 1: & & 4 & 36 & 236 & 1364 & 7368 & 38152 & 191908 \\
\hline 2: & . & . & 15 & 198 & 1723 & 12438 & 80628 & 487202 \\
\hline 3: & . & . & & 56 & 976 & 10576 & 91448 & 690904 \\
\hline 4: & . & . & & & 209 & 4527 & 58685 & 590894 \\
\hline 5: & . & . & . & & & 780 & 20196 & 304696 \\
\hline 6: & 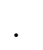 & . & & & & & 2911 & 87692 \\
\hline 7 & . & . & . & 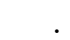 & & & . & 10864 \\
\hline
\end{tabular}

showing that $\Phi_{R}(x, y)$ should start as

$$
\Phi(x, y)=1+x y(5+4 y)+x^{2} y^{2}\left(22+36 y+15 y^{2}\right)+x^{3} y^{3}\left(95+236 y+198 y^{2}+56 y^{3}\right)+\ldots
$$

But note that the calculation of $\Phi_{R}(z, z)$ in the preceding section gives

$$
\Phi_{R}(z, z)=1+5 z^{2}+4 * z^{3}+22 z^{4}+36 z^{5}+110 z^{6}+236 z^{7}+607 z^{8}+1420 z^{9}+3483 z^{10}+\ldots
$$

and as you see this is not sufficient to get the preceding columns e.g. to find the decomposition $110=$ $15+95=\left|\operatorname{Tor}_{2,4}^{H K R}(k, k)\right|+\left|\operatorname{Tor}_{3,3}^{H K R}(k, k)\right|$ etc. But now we use the Löfwall formula (4) where we replace $x$ by $z^{u}$ for any integer $u \geq 1$ and $y$ by $z$ :

$$
1 / \Phi_{R}\left(z^{u}, z\right)=\left(1+1 / z^{u}\right) / H_{A}\left(z^{u}, z\right)-\left(1-H_{I / I^{2}}(z) z^{u}+H_{I^{2}}(z) z^{2 u}\right) z^{u}
$$

gives that $H_{A}\left(z^{u}, z\right)$ is the Hilbert series of a new variant of the algebra $A$ where all previous generators of degree $s$ have been replaced by generators of degree $s+u-1$. Now the Hilbertseries of $A$ with generators of these degrees can still be calculated with BERGMAN. We illustrate this with the case 46 just treated when $u=31$ The new input file for BERGMAN will therefore be:

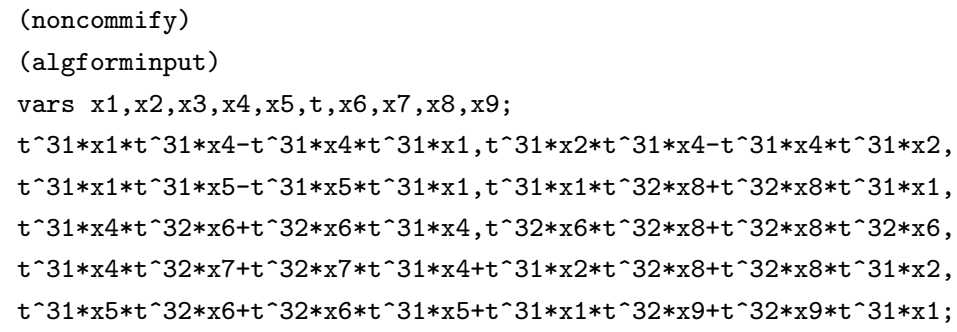

Note that here the generators still all have degree 1 and BERGMAN now gives for this infile the inverse of its Hilbert series:

$$
1-10 * z+3 z^{64}+4 z^{65}+z^{66}
$$

(the permutation $x 1, x 2, x 3, t, x 4, x 5, x 6, x 7, x 8, x 9$ still gives a finite Gröbner basis), and the Ufnarovskij method now still gives that $1 / A\left(z^{31}, z\right)=1+3 z^{64}+4 z^{65}+z^{66}-5 z^{32}-4 z^{33}$. On the other hand the Hilbert series of $H K R(x, y)=1+5 x y+4 x y^{2}+3 x^{2} y^{2}+4 x^{2} y^{3}+x^{2} y^{4}$ so that $H K R\left(-z^{31}, z\right)=1-5 z^{32}-4 z^{33}+$ $3 z^{64}+4 z^{65}+z^{66}$ and the formula (10) now also gives

$$
1 / \Phi_{R}\left(z^{31}, z\right)=\left(1+1 / z^{31}\right) / A\left(z^{31}, z\right)-H K R\left(-z^{31}, z\right) / z^{31}=1+3 z^{64}+4 z^{65}+z^{66}-5 z^{32}-4 z^{33}
$$

But the taylor series of $1 /\left(1+3 z^{64}+4 z^{65}+z^{66}-5 z^{32}-4 z^{33}\right)$ is

$$
\begin{gathered}
1+z^{32}(5+4 z)+z^{64}\left(22+36 z+15 z^{2}\right)+z^{96}\left(95+236 z+198 z^{2}+56 z^{3}\right)+ \\
+z^{128}\left(409+1364 z+1723 z^{2}+976 z^{3}+109 z^{4}\right)+z^{160}\left(1760+7368 z+12438 z^{2}+\ldots\right)+\ldots
\end{gathered}
$$

which "explains" the columns above at the beginning of this section and gives an indication that

$$
1 / \Phi_{R}(x, y)=1-\left(5+4 y+y^{2}\right) x y+\left(3+4 y+y^{2}\right) x^{2} y^{2}
$$

In a similar way one treats all the quadratic embedding dimension 4 cases and the results are given in the tables A-D that follow. 
Table A. Double Tor of HKR

\begin{tabular}{|c|c|c|}
\hline $\mathrm{R}$ & ideal gen:s (ex.) & 1/Double Tor of $H K R$ \\
\hline 1 & 0 & 1 \\
\hline 2 & $x^{2}$ & $1-x y$ \\
\hline 3 & $x^{2}, y^{2}$ & $1-2 x y+x^{2} y^{2}$ \\
\hline 4 & $x^{2}, x y$ & $1-(2+y) x y$ \\
\hline 5 & $x^{2}, y^{2}, z^{2}$ & $1-3 x y+3 x^{2} y^{2}-x^{3} y^{3}$ \\
\hline 6 & $x^{2}, y^{2}+x z, y z$ & $1-\left(3+y+2 y^{2}\right) x y+(3-y) x^{2} y^{2}-x^{3} y^{3}$ \\
\hline 7 & $x^{2}+y^{2}, z^{2}+u^{2}, x z+y u$ & $1-\left(3+2 y+4 y^{2}+y^{3}\right) x y+(3-y) x^{2} y^{2}-x^{3} y^{3}$ \\
\hline 8 & $x^{2}, y^{2}, x z$ & $1-(3+y) x y+(2+y) x^{2} y^{2}$ \\
\hline 9 & $x^{2}, x y, y^{2}$ & $1-(3+2 y) x y$ \\
\hline 10 & $x^{2}, x y, x z$ & $1-\left(3+3 y+y^{2}\right) x y$ \\
\hline 11 & $x^{2}, y^{2}, z^{2}, u^{2}$ & $1-4 x y+6 x^{2} y^{2}-4 x^{3} y^{3}+x^{4} y^{4}$ \\
\hline 12 & $x^{2}+x y, y^{2}+y u, x^{2}+x u, u^{2}+z u$ & $1-\left(4+y+3 y^{2}+3 y^{3}\right) x y+\left(6-y^{2}\right) x^{2} y^{2}-4 x^{3} y^{3}+x^{4} y^{4}$ \\
\hline 13 & $x^{2}+z^{2}+u^{2}, y^{2}, x z, y u+z u$ & $1-\left(4+y+2 y^{2}\right) x y+\left(6+2 y^{2}\right) x^{2} y^{2}-(4-y) x^{3} y^{3}+x^{4} y^{4}$ \\
\hline 14 & $x z, y^{2}, z^{2}+u^{2}, y u+z u$ & $1-\left(4+2 y+5 y^{2}\right) x y+\left(6-3 y+2 y^{2}\right) x^{2} y^{2}-(4-y) x^{3} y^{3}+x^{4} y^{4}$ \\
\hline 15 & $x z, y^{2}, y z+u^{2}, y u+z u$ & $1-\left(4+3 y+8 y^{2}+2 y^{3}\right) x y+(6-4 y) x^{2} y^{2}-(4-y) x^{3} y^{3}+x^{4} y^{4}$ \\
\hline 16 & $x y+z^{2}+y u, y^{2}, y u+z u, x z$ & $1-\left(4+4 y+10 y^{2}+3 y^{3}\right) x y+(6-4 y) x^{2} y^{2}-(4-y) x^{3} y^{3}+x^{4} y^{4}$ \\
\hline 17 & $x z, y z+x u, y^{2}, y u+z u$ & $\left(1-\left(4+y^{2}\right) x y+\left(6-4 y-6 y^{2}-5 y^{3}-y^{4}\right) x^{2} y^{2}-(4-y) x^{3} y^{3}+x^{4} y^{4}\right) /\left(1+x y^{2}\right)$ \\
\hline 18 & $x^{2}, y^{2}, z^{2}, y u$ & $1-(4+y) x y+(5+2 y) x^{2} y^{2}-(2+y) x^{3} y^{3}$ \\
\hline 19 & $x z, y^{2}, y u+z u, u^{2}$ & $1-\left(4+2 y+3 y^{2}+y^{3}\right) x y+\left(5-y^{2}\right) x^{2} y^{2}-(2+y) x^{3} y^{3}$ \\
\hline 20 & $x z, y^{2}, y u+z^{2}, y u+z u$ & $1-\left(4+3 y+5 y^{2}+2 y^{3}\right) x y+(5+2 y) x^{2} y^{2}-(2+y) x^{3} y^{3}$ \\
\hline 21 & $x z, y^{2}, z^{2}, y u+z u$ & $1-(4+2 y) x y+\left(4+4 y+y^{2}\right) x^{2} y^{2}$ \\
\hline 22 & $x^{2}+x y, x u, x z+y u, y^{2}$ & $1-(4+y) x y+\left(4+4 y-2 y^{3}-y^{4}\right) x^{2} y^{2}-\left(1+4 y+y^{2}\right) x^{3} y^{4}+\left(-1-2 y+y^{2}\right) x^{4} y^{4}$ \\
\hline 23 & $x z, x u, y^{2}, z^{2}$ & $1-(4+2 y) x y+(3+2 y) x^{2} y^{2}$ \\
\hline 24 & $x z, y^{2}, y z+z^{2}, y u+z u$ & $1-(4+y) x y+(5+2 y) x^{2} y^{2}-(2+y) x^{3} y^{3}$ \\
\hline 25 & $x^{2}, x y, x z, u^{2}$ & $1-\left(4+3 y+y^{2}\right) x y+\left(3+3 y+y^{2}\right) x^{2} y^{2}$ \\
\hline 26 & $x z, y^{2}, y u, z u$ & $1-(4+3 y) x y+(1+y) x^{2} y^{2}$ \\
\hline 27 & $x y, x z, y^{2}, y z$ & $1-\left(4+4 y+y^{2}\right) x y$ \\
\hline 28 & $x^{2}, x y, x z, x u$ & $1-(4+y) x y+(5+2 y) x^{2} y^{2}-(2+y) x^{3} y^{3}$ \\
\hline 29 & $x^{2}+x y, y^{2}+x u, z^{2}+x u, z u+u^{2}$ & $1-\left(5+5 y+16 y^{2}+5 y^{3}\right) x y+(10-10 y) x^{2} y^{2}-(10-5 y) x^{3} y^{3}+(5-y) x^{4} y^{4}-x^{5} y^{5}$ \\
\hline 30 & $x y+u^{2}, x z, x^{2}+z^{2}+u^{2}, y^{2}, y u+z u$ & $\frac{1-\left(5+y+5 y^{2}\right) x y+\left(10-10 y-10 y^{2}-10 y^{3}-y^{4}\right) x^{2} y^{2}-\left(10-5 y-y^{3}\right) x^{3} y^{3}+(5-y) x^{4} y^{4}-x^{5} y^{5}}{1+x y^{2}}$ \\
\hline 31 & $\begin{array}{l}x^{2}-y^{2}, y^{2}-z^{2}, z^{2}-u^{2}, x z+y u \\
-x^{2}+x y-y z+x u\end{array}$ & $\frac{1-\left(5+2 y+8 y^{2}+3 y^{3}\right) x y+\left(10-10 y-12 y^{2}-13 y^{3}-4 y^{4}\right) x^{2} y^{2}-(10-5 y) x^{3} y^{3}+(5-y) x^{4} y^{4}-x^{5} y^{5}}{1+x y^{2}}$ \\
\hline 32 & $x^{2}+z^{2}, x z, y^{2}, y u+z u, u^{2}$ & $1-\left(5+3 y+7 y^{2}+2 y^{3}\right) x y+\left(9-3 y-2 y^{2}\right) x^{2} y^{2}-\left(7+y-y^{2}\right) x^{3} y^{3}+(2+y) x^{4} y^{4}$ \\
\hline
\end{tabular}


Table B. Double Tor of HKR

\begin{tabular}{|c|c|c|}
\hline $\mathrm{R}$ & ideal gen:s (ex.) & 1/Double Tor of $H K R$ \\
\hline 33 & $\begin{array}{l}x^{2}+x y, y^{2}+y z \\
y^{2}+x u, z^{2}+x u, z u+u^{2}\end{array}$ & $1-\left(5+4 y+10 y^{2}+4 y^{3}\right) x y+\left(9-4 y-3 y^{2}\right) x^{2} y^{2}-\left(7+y-y^{2}\right) x^{3} y^{3}+(2+y) x^{4} y^{4}$ \\
\hline 34 & $\begin{array}{l}x^{2}+x y+y u+u^{2}, y^{2}, x z \\
x^{2}+z^{2}+u^{2}, y u+z u\end{array}$ & $\begin{array}{l}\left(1-5 x y+\left(9-5 y-18 y^{2}-15 y^{3}-4 y^{4}\right) x^{2} y^{2}-\left(5+5 y+9 y^{3}+10 y^{4}+3 y^{5}\right) x^{3} y^{3}+\right. \\
\left.+(-5+9 y) x^{4} y^{4}+(9-5 y) x^{5} y^{5}-(5-y) x^{6} y^{6}+x^{7} y^{7}\right) /\left(1+x y^{2}\right)^{2}\end{array}$ \\
\hline 35 & $\begin{array}{l}x^{2}+z^{2}+u^{2}, y^{2}, x z \\
x y+y z+y u, y u+z u\end{array}$ & $\begin{array}{l}\left(1-\left(5+y+2 y^{2}+y^{3}\right) x y+\left(9-4 y-12 y^{2}-10 y^{3}-3 y^{4}\right) x^{2} y^{2}-\left(7+y-y^{2}\right) x^{3} y^{3}+\right. \\
\left.+(2+y) x^{4} y^{4}\right) /\left(1+x y^{2}\right)\end{array}$ \\
\hline 36 & $\begin{array}{l}x^{2}+y^{2}, z^{2}, u^{2} \\
y z-y u, x z+z u\end{array}$ & $1-\left(5+3 y+4 y^{2}+2 y^{3}\right) x y+\left(8+2 y-3 y^{2}-y^{3}\right) x^{2} y^{2}-\left(4+4 y+y^{2}\right) x^{3} y^{3}$ \\
\hline 37 & $\begin{array}{l}x^{2}, y^{2}, x y-z u \\
y z-x u,(x-y)(z-u)\end{array}$ & $\begin{array}{l}\left(1-\left(5+2 y+4 y^{2}+2 y^{3}\right) x y+\left(8-3 y-7 y^{2}-7 y^{3}-3 y^{4}\right) x^{2} y^{2}-\left(4-2 y+2 y^{2}+3 y^{3}\right) x^{3} y^{3}+\right. \\
\left.+\left(-1-4 y-y^{2}+y^{3}\right) x^{4} y^{4}+\left(1+2 y+y^{2}\right) x^{5} y^{5}\right) /\left(1+x y^{2}\right)\end{array}$ \\
\hline 38 & $x^{2}, y^{2}, z^{2}, z u, u^{2}$ & $1-(5+2 y) x y+(7+4 y) x^{2} y^{2}-(3+2 y) x^{3} y^{3}$ \\
\hline 39 & $\begin{array}{l}x^{2}+y z+u^{2}, x z+z^{2}+y u \\
x y, x u, z u\end{array}$ & $1-(5+3 y+3 y+y) x y+(7+2 y-y) x^{2} y^{2}-(3+2 y) x^{3} y^{3}$ \\
\hline 40 & $\begin{array}{l}x^{2}-x u, x u-y^{2}, y^{2}-z^{2} \\
z^{2}-u^{2}, x z-y u\end{array}$ & $1-\left(5+4 y+6 y^{2}+3 y^{3}\right) x y+\left(7+y-2 y^{2}\right) x^{2} y^{2}-(3+2 y) x^{3} y^{3}$ \\
\hline 41 & $x y, y^{2}, z^{2}, z u, u^{2}$ & $1-(5+3 y) x y+\left(6+7 y+2 y^{2}\right) x^{2} y^{2}$ \\
\hline 42 & $\begin{array}{l}x^{2}+x y, z u, y^{2} \\
x u, x z+y u\end{array}$ & $\begin{array}{l}\left(1-(5+2 y) x y+\left(6+y-5 y^{2}-5 y^{3}-2 y^{4}\right) x^{2} y^{2}+\right. \\
\left.+\left(-1+y-y^{3}\right) x^{3} y^{3}+\left(-1-2 y-y^{2}\right) x^{4} y^{4}\right) /\left(1+x y^{2}\right)\end{array}$ \\
\hline 43 & $x^{2}, y^{2}, y z, z u, u^{2}$ & $1-(5+3 y) x y+(5+4 y) x^{2} y^{2}-(1+y) x^{3} y^{3}$ \\
\hline 44 & $\begin{array}{l}x z, y z, y^{2} \\
y u+z u, z^{2}+u^{2}\end{array}$ & $1-\left(5+4 y+3 y^{2}+2 y^{3}\right) x y+\left(5+3 y-y^{2}\right) x^{2} y^{2}-(1+y) x^{3} y^{3}$ \\
\hline 45 & $\begin{array}{l}x y+y z, x y+z^{2}+y u \\
y u+z u, y^{2}, x z\end{array}$ & $1-(5+4 y) x y+\left(4+6 y+2 y^{2}\right) x^{2} y^{2}$ \\
\hline 46 & $x^{2}, x y, y z, z u, u^{2}$ & $1-(5+4 y) x y+\left(3+4 y+y^{2}\right) x^{2} y^{2}$ \\
\hline $\begin{array}{l}46 \\
\text { va }\end{array}$ & $\begin{array}{l}x z+u^{2}, x y, x u, x^{2} \\
z u+y^{2}+z^{2}\end{array}$ & $1-\left(5+4 y+y^{2}\right) x y+\left(4+4 y+y^{2}\right) x^{2} y^{2}$ \\
\hline 47 & $\begin{array}{l}x^{2}+x y, y^{2}, x u \\
x z+y u,-x^{2}+x z-y z\end{array}$ & $1-\left(5+5 y+2 y^{2}+y^{3}\right) x y+\left(3+4 y+y^{2}\right) x^{2} y^{2}$ \\
\hline 48 & $\begin{array}{l}x y, z^{2}+y u, y u+z u \\
y^{2}, x z\end{array}$ & $1-\left(5+5 y+3 y^{2}+2 y^{3}\right) x y+\left(3+2 y-y^{2}\right) x^{2} y^{2}-(1+y) x^{3} y^{3}$ \\
\hline 49 & $x z, y^{2}, z^{2}, y u, z u$ & $1-\left(5+5 y+y^{2}\right) x y+\left(2+3 y+y^{2}\right) x^{2} y^{2}$ \\
\hline 50 & $x^{2}, x y, x z, y^{2}, z^{2}$ & $1-\left(5+5 y+y^{2}\right) x y+(1+y) x^{2} y^{2}$ \\
\hline 51 & $x y, x z, y z+x u, z^{2}, z u$ & $1-\left(5+6 y+3 y^{2}+y^{3}\right) x y+(1+y) x^{2} y^{2}$ \\
\hline 52 & $x^{2}, x y, x z, y^{2}, y z$ & $1-\left(5+6 y+2 y^{2}\right) x y$ \\
\hline 53 & $y^{2}-u^{2}, x z, y z, z^{2}, z u$ & $1-\left(5+7 y+4 y^{2}+y^{3}\right) x y$ \\
\hline 54 & $x^{2}, x z, y^{2}, z^{2}, y u+z u, u^{2}$ & $1-(6+4 y) x y+\left(9+12 y+4 y^{2}\right) x^{2} y^{2}$ \\
\hline 55 & $\begin{array}{l}x^{2}+x y, x z+y u, x u \\
y^{2}, z^{2}, z u+u^{2}\end{array}$ & $\begin{array}{l}\left(1-(6+2 y) x y+\left(9-y-9 y^{2}-9 y^{3}-4 y^{4}\right) x^{2} y^{2}-\left(1-6 y+7 y^{2}+18 y^{3}+12 y^{4}+4 y^{5}\right) x^{3} y^{3}+\right. \\
\left.+\left(-6-13 y-3 y^{2}+3 y^{3}\right) x^{4} y^{4}+\left(3+6 y+3 y^{2}\right) x^{5} y^{5}\right) /\left(1+x y^{2}\right)^{2}\end{array}$ \\
\hline
\end{tabular}


Table C. Double Tor of HKR

\begin{tabular}{|c|c|c|}
\hline $\mathrm{R}$ & ideal gen:s (ex.) & 1/Double Tor of $H K R$ \\
\hline 56 & $\begin{array}{l}x^{2}+x z+u^{2}, x y, x u \\
x^{2}-y^{2}, z^{2}, z u\end{array}$ & $\begin{array}{l}\left(1-\left(6+3 y+y^{2}+y^{3}\right) x y+\left(9+y-11 y^{2}-10 y^{3}-4 y^{4}\right) x^{2} y^{2}+\right. \\
\left.\left.+\left(-4-y+y^{2}-y^{3}\right) x^{3} y^{3}-(1+y) x^{4} y^{5}\right)\right) /\left(1+x y^{2}\right)\end{array}$ \\
\hline 57 & $x^{2}+y z+u^{2}, x y, x u, z u, y^{2}+z^{2}, x z+y u$ & $1-\left(6+5 y+4 y^{2}+3 y^{3}\right) x y+\left(8+5 y-3 y^{2}-y^{3}\right) x^{2} y^{2}-\left(3+4 y+y^{2}\right) z^{3} y^{3}$ \\
\hline $\begin{array}{l}57 \\
\mathrm{v} 2\end{array}$ & $\begin{array}{l}x^{2}+y^{2}+z^{2}, x y \\
x u, y z, z u, x z+u^{2}\end{array}$ & $1-\left(6+5 y+5 y^{2}+2 y^{3}\right) x y+\left(9+4 y-2 y^{2}-y^{3}\right) x^{2} y^{2}-\left(4+4 y+y^{2}\right) z^{3} y^{3}$ \\
\hline 58 & $x y, x^{2}+z u, y^{2}, z^{2}, y u+x z, x u$ & $1-(6+5 y) x y+\left(6+9 y+3 y^{2}\right) x^{2} y^{2}$ \\
\hline 59 & $\begin{array}{l}x^{2}-y^{2}, x y, x u \\
z^{2}, z u, x z+y u\end{array}$ & $\begin{array}{l}\left(1-(6+4 y) x y+\left(6+3 y-3 y^{2}-3 y^{3}-2 y^{4}\right) x^{2} y^{2}+\right. \\
\left.+\left(4+2 y-3 y^{2}-y^{3}\right) x^{3} y^{4}-\left(1+4 y+4 y^{2}+y^{3}\right) x^{4} y^{4}\right) /\left(1+x y^{2}\right)\end{array}$ \\
\hline $\begin{array}{l}59 \\
\text { va }\end{array}$ & $\begin{array}{l}x^{2}-y^{2}, x y, y z \\
z u, x z+u^{2}, x u\end{array}$ & $\begin{array}{l}\left(1-\left(6+4 y+y^{2}\right) x y+\left(7+3 y-3 y^{2}-3 y^{3}-y^{4}\right) x^{2} y^{2}+\right. \\
\left.+\left(4+2 y-2 y^{2}-y^{3}\right) x^{3} y^{4}-\left(2+5 y+4 y^{2}+y^{3}\right) x^{4} y^{4}\right) /\left(1+x y^{2}\right)\end{array}$ \\
\hline $\begin{array}{l}60 \\
\text { va }\end{array}$ & $\begin{array}{l}x^{2}+y z+u^{2}, x y, z u \\
z^{2}, x z+y u, x u\end{array}$ & $\begin{array}{l}\left(1-(6+4 y) x y+\left(6+2 y-5 y^{2}-4 y^{3}-2 y^{4}\right) x^{2} y^{2}+\right. \\
\left.+\left(-1+3 y+4 y^{2}-y^{3}-y^{4}\right) x^{3} y^{3}-\left(1+2 y+y^{2}\right) x^{4} y^{5}\right) /\left(1+x y^{2}\right)\end{array}$ \\
\hline 61 & $x^{2}-y^{2}, x y, z^{2}, x u, z u, u^{2}$ & $1-\left(6+5 y+y^{2}\right) x y+\left(6+6 y+y^{2}\right) x^{2} y^{2}-(y+1) x^{3} y^{3}$ \\
\hline 62 & $x^{2}-y^{2}, x y, x u, y z+y u, z^{2}, z u$ & $1-\left(6+6 y+3 y^{2}+3 y^{3}\right) x y+\left(5+6 y-y^{2}\right) x^{2} y^{2}-x^{3} y^{4}$ \\
\hline $\begin{array}{l}62 \\
\text { va }\end{array}$ & $\begin{array}{l}x^{2}+y z+u^{2}, y u \\
z u, x y, z^{2}, x u\end{array}$ & $1-\left(6+6 y+4 y^{2}+2 y^{3}\right) x y+(6+5 y) x^{2} y^{2}-(1+y) x^{3} y^{3}$ \\
\hline 63 & $x^{2}, x y, x u, y^{2}, z^{2}, z u$ & $1-\left(6+6 y+y^{2}\right) x y+\left(4+6 y+2 y^{2}\right) x^{2} y^{2}$ \\
\hline $\begin{array}{l}63 \\
\mathrm{v} 4\end{array}$ & $y^{2}, x z+y u, z u, x y, z^{2}, x u$ & $1-(6+6 y) x y+\left(3+6 y+2 y^{2}\right) x^{2} y^{2}$ \\
\hline $\begin{array}{l}63 \\
\mathrm{v} 8\end{array}$ & $\begin{array}{l}x^{2}, x y, x u, y u, z^{2} \\
x z+u^{2}, y^{2}+z^{2}+z u\end{array}$ & $1-\left(6+6 y+2 y^{2}\right) x y+\left(5+6 y+2 y^{2}\right) x^{2} y^{2}$ \\
\hline $\begin{array}{l}63 \\
\text { ne }\end{array}$ & $\begin{array}{l}x^{2}, x y, x z+u^{2}, x u \\
y^{2}+z^{2}, z u\end{array}$ & $\begin{array}{l}\left(1-\left(6+5 y+y^{2}\right) x y+\left(4-4 y^{2}-2 y^{3}-y^{4}\right) x^{2} y^{2}+\right. \\
\left.+\left(4+5 y+y^{2}\right) x^{3} y^{4}\right) /\left(1+x y^{2}\right)\end{array}$ \\
\hline 64 & $x^{2}-y^{2}, x y, z^{2}, x u, y u, z u$ & $1-\left(6+6 y+y^{2}\right) x y+(3+3 y) x^{2} y^{2}-(1+y) x^{3} y^{3}$ \\
\hline 65 & $\begin{array}{l}x^{2}, x y, x z, y^{2}, y u+z^{2}, y u+z u \\
x^{2}, x y, x z, y^{2}, y u+z^{2}, y u+z u\end{array}$ & $\begin{array}{l}1-\left(6+7 y+4 y^{2}+2 y^{3}\right) x y+ \\
+\left(3+2 y-y^{2}\right) x^{2} y^{2}-(1+y) x^{3} y^{3}\end{array}$ \\
\hline 66 & $x z, y^{2}, y u, z^{2}, z u, u^{2}$ & $1-\left(6+7 y+2 y^{2}\right) x y+\left(2+3 y+y^{2}\right) x^{2} y^{2}$ \\
\hline $\begin{array}{l}66 \\
\mathrm{v} 5\end{array}$ & $x y, x z+u^{2}, x u, y u, z u, z^{2}$ & $1-\left(6+7 y+y^{2}\right) x y+\left(1+3 y+y^{2}\right) x^{2} y^{2}$ \\
\hline 67 & $x y, x z, y^{2}, y u, z^{2}, z u$ & $1-\left(6+8 y+3 y^{2}\right) x y+\left(1+2 y+y^{2}\right) x^{2} y^{2}$ \\
\hline 68 & $x^{2}, x y, x z, y^{2}, y z, z^{2}$ & $1-\left(6+8 y+3 y^{2}\right) x y$ \\
\hline $68 v$ & $x^{2}, x y, x z, x u, u^{2}, y^{2}+z^{2}+z u$ & $1-\left(6+8 y+4 y^{2}+y^{3}\right) x y+(1+y) x^{2} y^{2}$ \\
\hline 69 & $x^{2}, x z, x u, x y-z u, y z, z^{2}$ & $1-\left(6+9 y+5 y^{2}+y^{3}\right) x y$ \\
\hline 70 & $x^{2}, x y, x z, x u, y^{2}, y z$ & $1-\left(6+9 y+5 y^{2}+y^{3}\right) x y$ \\
\hline 71 & $x^{2}, y^{2}, z^{2}, u^{2}, x y, z u, y z+x u$ & $1-\left(7+8 y+2 y^{2}\right) x y+\left(5+8 y+3 y^{2}\right) x^{2} y^{2}$ \\
\hline
\end{tabular}


Table D. Double Tor of HKR

\begin{tabular}{|c|c|c|}
\hline $\mathrm{R}$ & ideal gen:s (ex.) & 1/Double Tor of $H K R$ \\
\hline $\begin{array}{r}71 \\
\mathrm{v} 16\end{array}$ & $\begin{array}{l}x^{2}, y^{2}+z^{2}, x y, y z \\
z u, x z+u^{2}, x u\end{array}$ & $\begin{array}{l}\left(1-\left(7+7 y+2 y^{2}\right) x y+\left(5+y-3 y^{3}-y^{4}\right) x^{2} y^{2}+\right. \\
\left.+\left(5+7 y+2 y^{2}\right) x^{3} y^{4}\right) /\left(1+x y^{2}\right)\end{array}$ \\
\hline $\begin{array}{l}71 \\
\mathrm{v} 4\end{array}$ & $x^{2}+u^{2}, x y, x u, y^{2}, y z, z^{2}, z u$ & $1-\left(7+8 y+3 y^{2}\right) x y+\left(6+8 y+3 y^{3}\right) x^{2} y^{2}$ \\
\hline $\begin{array}{l}71 \\
\text { v7 }\end{array}$ & $x^{2}, y^{2}, z^{2}, x z+u^{2}, x u, y z, z u$ & $1-\left(7+8 y+y^{2}\right) x y+\left(4+8 y+3 y^{3}\right) x^{2} y^{2}$ \\
\hline $\begin{array}{l}71 \\
\mathrm{v} 5\end{array}$ & $\begin{array}{l}x^{2}+x y, x^{2}+y z, x y+y^{2}, z^{2} \\
z^{2}, x u, z u, x z+u^{2}\end{array}$ & $1-(7+8 y) x y+\left(3+8 y+3 y^{3}\right) x^{2} y^{2}$ \\
\hline 72 & $x^{2}-y^{2}, z^{2}, x y, y z, z u, x u$ & $1-\left(7+9 y+2 y^{2}\right) x y+\left(2+5 y+2 y^{3}\right) x^{2} y^{2}$ \\
\hline $\begin{array}{l}72 \\
\mathrm{v} 1\end{array}$ & $\begin{array}{l}x u+u^{2}, x^{2}+x y, y^{2}+x u, y^{2}+y z \\
y^{2}+y z, y u+z u, z^{2}+x u, z u+u^{2}\end{array}$ & $1-\left(7+9 y+3 y^{2}\right) x y+\left(3+5 y+2 y^{3}\right) x^{2} y^{2}$ \\
\hline $\begin{array}{r}72 \\
\mathrm{v} 2 \mathrm{e}\end{array}$ & $\begin{array}{l}y z, x^{2}+x y, x z+y u, x u, z^{2} \\
z u, x^{2}+u^{2}\end{array}$ & $1-\left(7+9 y+1 y^{2}\right) x y+\left(1+5 y+2 y^{3}\right) x^{2} y^{2}$ \\
\hline 73 & $x^{2}, y^{2}, z^{2}, u^{2}, x u, y u, z u$ & $\begin{array}{l}1-\left(7+9 y+4 y^{2}+y^{3}\right) x y+ \\
+(3+3 y) x^{2} y^{2}-(1+y) x^{3} y^{3}\end{array}$ \\
\hline 74 & $x^{2}, x y+z^{2}, y z, x u, y u, z u, u^{2}$ & $\begin{array}{l}1-\left(7+10 y+7 y^{2}+3 y^{3}\right) x y+ \\
+\left(3+2 y-y^{2}\right) x^{2} y^{2}-(1+y) x^{3} y^{3}\end{array}$ \\
\hline 75 & $x^{2}, x y, x z, y^{2}, y z, y u, u^{2}$ & $1-\left(7+10 y+5 y^{2}+y^{3}\right) x y+\left(2+3 y+y^{2}\right) x^{2} y^{2}$ \\
\hline $\begin{array}{l}75 \\
\mathrm{v} 1\end{array}$ & $y^{2}, x z, y z+x u, z^{2}, y u, z u, u^{2}$ & $1-\left(7+10 y+4 y^{2}\right) x y+\left(1+2 y+y^{2}\right) x^{2} y^{2}$ \\
\hline $\begin{array}{l}75 \\
\mathrm{v} 2\end{array}$ & $x y, x z+u^{2}, x u, y z+u^{2}, y u, z u, z^{2}$ & $1-\left(7+10 y+3 y^{2}\right) x y+\left(2 y+y^{2}\right) x^{2} y^{2}$ \\
\hline 76 & $x^{2}, x y, x z, x u, z^{2}, z u, y u$ & $1-\left(7+11 y+6 y^{2}+y^{3}\right) x y$ \\
\hline 77 & $x^{2}, x y, x z, x u, y^{2}, y z, y u$ & $1-\left(7+12 y+8 y^{2}+2 y^{3}\right) x y$ \\
\hline 78 & $x^{2}, x y, y^{2}, z^{2}, z u, u^{2}, x z+y u, y z-x u$ & $1-\left(8+12 y+5 y^{2}\right) x y+\left(2+4 y+y^{2}\right) x^{2} y^{2}$ \\
\hline $\begin{array}{l}78 \\
\mathrm{v} 1\end{array}$ & $x^{2}, y^{2}, z^{2}, u^{2}, x y, x z, x u, y u$ & $1-\left(8+12 y+6 y^{2}+y^{3}\right) x y+\left(3+5 y+2 y^{2}\right) x^{2} y^{2}$ \\
\hline $\begin{array}{r}78 \\
\mathrm{v} 2 \mathrm{e} \\
\end{array}$ & $x u, y u+x z, y z, x^{2}, y^{2}, z^{2}+x y, u^{2}, z u$ & $1-\left(8+12 y+3 y^{2}\right) x y+\left(4 y+2 y^{2}\right) x^{2} y^{2}$ \\
\hline $\begin{array}{r}78 \\
\mathrm{v} 3 \mathrm{v}\end{array}$ & $x u, y u+x z, y z, x^{2}, y^{2}, z^{2}+x z, u^{2}, z u$ & $1-\left(8+12 y+4 y^{2}\right) x y+\left(1+4 y+2 y^{2}\right) x^{2} y^{2}$ \\
\hline 79 & $x^{2}, x y, x z, x u, y^{2}, y u, z^{2}, z u$ & $1-\left(8+13 y+7 y^{2}+y^{3}\right) x y+\left(1+2 y+y^{2}\right) x^{2} y^{2}$ \\
\hline 80 & $x^{2}, x y, x z, y z, y^{2}, y u, z^{2}, z u$ & $1-\left(8+14 y+9 y^{2}+2 y^{3}\right) x y$ \\
\hline 81 & $x^{2}, y^{2}, z^{2}, u^{2}, x y, x z, y z-x u, y u, z u$ & $1-\left(9+16 y+9 y^{2}\right) x y+x^{2} y^{4}$ \\
\hline $\begin{array}{l}81 \\
\text { va }\end{array}$ & $x^{2}, y^{2}, z^{2}, u^{2}, x y, x z, x u, y u, z u$ & $1-\left(9+16 y+10 y^{2}+2 y^{3}\right) x y+\left(1+2 y+y^{2}\right) x^{2} y^{2}$ \\
\hline 82 & $x^{2}, x y, x z, x u, y^{2}, z u, u^{2}, y z, y u$ & $1-\left(9+17 y+12 y^{2}+3 y^{3}\right) x y$ \\
\hline 83 & $x^{2}, y^{2}, z^{2}, u^{2}, x y, x z, x u, y z, y u, z u$ & $1-\left(10+20 y+15 y^{2}+4 y^{3}\right) x y$ \\
\hline
\end{tabular}

Case 81 is Gorenstein 


\section{Higher embedding dimensions. Three examples.}

Many new phenomena come up in connection with higher embedding dimensions of local rings. Here we will just mention three very different cases of $R=k[x, y, z, u, v] / J$ where the $J$ is an ideal generated by quadratic forms in $x, y, z, u, v$

Case I $R_{I}=k[x, y, z, u, v] /\left(y^{2}, u^{2}, y z+x u, z u+y v, z^{2}-y u-x v\right)$

Case II $R_{I I}=k[x, y, z, u, v] /\left(x u-x v, x v-z u, y v, u^{2}, v^{2}\right)$

Case III $R_{I I I}=k[x, y, z, u, v] /\left(x z+y u, y v, z u+u v, z^{2}, v^{2}\right)$

In all these three cases the resolution of $\mathrm{J}$ over $k[x, y, z, u, v]$ has the same diagram of Betti numbers:

$$
\left(\begin{array}{cccccc}
1 & . & . & . & \cdot & . \\
\cdot & 5 & 3 & . & . & . \\
\cdot & . & 8 & 12 & 6 & 1
\end{array}\right)
$$

But the homological properties of $H K R$ and $R$ are very different:

Case I This is the case that Löfwall and I studied in [LR2], which also solved an old problem (the "Opera case"). In [LR2] we proved that the Hilbert series of $R^{!}$is transcendental and different for all values of the characteristic of the field $k$. Similar results are true for $H K R^{!}$and the Avramov spectral sequence does not degenerate. We will just give a few indications below.

Case II In [R1] I found, inspired by Lemaire [LE], a case of a local ring $(R, m)$ where the Yoneda Ext-algebra $\operatorname{Ext}_{R}^{*}(k, k)$ was not finitely generated. Furthermore that $R^{!}$had global dimension 3 . This is a variant of that example and similar assertions are true for $H K R$. Furthermore the Avramov spectral sequence degenerates.

Case III This is a variant of the first known example due to Anick [A] (cf also [LR1]) where $P_{R}(z)$ is transcendental. Here $H K R$ seems to have the same properties and the Avramov spectral sequence degenerates at least up to degrees 20.

More details: Case I.

We apply as before DGAlgebras and obtain that $H K R$ has the cube of the augmmentation ideal equal to 0 . Furthermore $H K R$ has 9 generators: five $X_{1}, X_{2}, X_{3}, X_{4}, X_{5}$ of degree $(1,2)$, three generators $X_{6}, X_{7}, X_{8}$ of degree $(2,3)$ and one $X_{9}$ of degree $(5,7)$. Furthermore these generators only satisfy 17 quadratic relations of which the following 9 are non-monomial relations:

$$
\begin{gathered}
X_{3} X_{4}-X_{2} X_{5}, X_{1} X_{4}-X_{3} X_{5}, X_{2} X_{3}+X_{1} X_{5}, X_{4} X_{7}-X_{5} X_{8}, X_{5} X_{6}+X_{3} X_{8}, \\
X_{4} X_{6}-X_{5} X_{7}+X_{2} X_{8}, X_{3} X_{6}+X_{1} X_{7}, X_{2} X_{6}+X_{3} X_{7}+X_{1} X_{8}, X_{7} X_{7}+2 X_{6} X_{8}
\end{gathered}
$$

and since the following 11 quadratic monomials do not occur among the 17 quadratic relations

$$
X_{1} X_{2}, X_{1} X_{3}, X_{2} X_{4}, X_{4} X_{5}, X_{1} X_{6}, X_{2} X_{7}, X_{4} X_{8}, X_{6} X_{6}, X_{6} X_{7}, X_{7} X_{8}, X_{8} X_{8}
$$

it follows that $H K R^{!}$is the quotient of the free algebra on the 9 generators $Y_{i}$ (which are dual to the $X_{i}$ ) by the two-sided ideal generated by the 21 quadratic relations:

$$
\begin{gathered}
{\left[Y_{1}, Y_{2}\right],\left[Y_{1}, Y_{3}\right],\left[Y_{2}, Y_{4}\right],\left[Y_{4}, Y_{5}\right],\left[Y_{1}, Y_{6}\right],\left[Y_{2}, Y_{7}\right],\left[Y_{4}, Y_{8}\right], Y_{6} Y_{6},\left[Y_{6}, Y_{7}\right],\left[Y_{7}, Y_{8}\right], Y_{8} Y_{8},} \\
{\left[Y_{1}, Y_{4}\right]+\left[Y_{3}, Y_{5}\right],\left[Y_{2}, Y_{3}\right]-\left[Y_{1}, Y_{5}\right],\left[Y_{3}, Y_{4}\right]+\left[Y_{2}, Y_{5}\right],\left[Y_{2}, Y_{6}\right]-\left[Y_{3}, Y_{7}\right],} \\
{\left[Y_{3}, Y_{7}\right]-\left[Y_{1}, Y_{8}\right],\left[Y_{3}, Y_{6}\right]-\left[Y_{1}, Y_{7}\right],\left[Y_{4}, Y_{6}\right]+\left[Y_{5}, Y_{7}\right],\left[Y_{5}, Y_{7}\right]+\left[Y_{2}, Y_{8}\right],} \\
{\left[Y_{5}, Y_{6}\right]-\left[Y_{3}, Y_{8}\right],\left[Y_{4}, Y_{7}\right]+\left[Y_{5}, Y_{8}\right],\left[Y_{7}, Y_{7}\right]-\left[Y_{6}, Y_{8}\right]}
\end{gathered}
$$

Here the graded commutators are as before and adding as before a new variable T giving a new algebra $A$ generated in degree 1 such that

$$
\frac{1}{H K R^{!}(z)}=\frac{1}{A(z)}+10 z-5 z^{2}-3 z^{3}-z^{6}
$$


We find using BERGMAN that

$$
\begin{aligned}
\frac{1}{A(z)} & =1-10 z+7 z^{4}+10 z^{5}+5 z^{6}-10 z^{8}-27 z^{9}-25 z^{10}+3 z^{11}+19 z^{12}+27 z^{13}+56 z^{14}+57 z^{15}+ \\
& +z^{16}-42 z^{17}-58 z^{18}-101 z^{19}-124 z^{20}-51 z^{21}+48 z^{22}+97 z^{23}+154 z^{24}+204 z^{25}+\ldots
\end{aligned}
$$

This gives $\frac{1}{H K R^{!}(z)}$ and since the two-variable Hilbert series of $H K R$ is $1+5 x y+3 x y^{2}+x y^{5}+7 x^{2} y^{2}+$ $10 x^{2} y^{3}+5 x^{2} y^{4}$ and therefore the one variable series with a minus sign in the first variable is $h=1-5 z^{2}-$ $3 z^{3}+7 z^{4}+10 z^{5}+4 z^{6}$, the formula

$$
\frac{1}{P_{H K R}(z)}=(1+1 / z) / H K R^{!}(z)-h / z
$$

gives

$$
P_{H K R}(z)=1+5 z^{2}+3 z^{3}+18 z^{4}+20 z^{5}+60 z^{6}+93 z^{7}+221 z^{8}+415 z^{9}+929 z^{10}+1936 z^{11}+\ldots
$$

whereas

$$
\frac{P_{R}(z)}{(1+z)^{5}}=1+5 z^{2}+3 z^{3}+18 z^{4}+20 z^{5}+59 z^{6}+92 z^{7}+216 z^{8}+407 z^{9}+907 z^{10}+1897 z^{11}+\ldots
$$

and they start differing in degree 6 so that the Avramov spectral sequence does not degenerate. The preceding calculations are in characteristic 0 . But e.g. in characteristic 7 we have

$$
\frac{P_{R}(z)}{(1+z)^{5}}=1+5 z^{2}+3 z^{3}+18 z^{4}+20 z^{5}+60 z^{6}+93 z^{7}+221 z^{8}+415 z^{9}+928 z^{10}+1935 z^{11}+\ldots
$$

In characteristic 7 we also have

$$
P_{H K R}(z)=1+5 z^{2}+3 z^{3}+18 z^{4}+20 z^{5}+60 z^{6}+93 z^{7}+221 z^{8}+415 z^{9}+929 z^{10}+1936 z^{11}+\ldots
$$

and these two series differ in degree 11, so that the Avramov spectral sequence does not degenerate.

Similar results are true in characteristic $2,3,5,11,13$, etc. But characteristic 2 is slightly different.

Important observation: The relations between the first 5 variables $X_{i}$ in $H K R$ are the same in all characteristic:

$$
X_{3} X_{4}-X_{2} X_{5}, X_{1} X_{4}-X_{3} X_{5}, X_{2} X_{3}+X_{1} X_{5}
$$

But the quotient of the exterior algebra in five variables with these 3 relations has a homology with transcendental Hilbert series which is different for all characteristic (it is the "skew" counterpart of the present Case I for ordinary polyomial rings). It occurs among the relations that Eisenbud and Koh have classified in $[\mathrm{Ei}-\mathrm{K}]$.

More details: Case II. Now the ring is $R=k[x, y, z, u, v] /\left(x u-x v, x v-z u, y v, u^{2}, v^{2}\right)$. We have that the Hilbert series $R^{!}(z)=\frac{(1+z)^{3}(1-z)}{\left(1-z-z^{2}\right)^{3}}$ and $R(z)=\frac{1+2 z-2 z^{2}-3 z^{3}+4 z^{4}-z^{5}}{(1-z)^{3}}$ and

$$
1 / P_{R}(z)=(1+1 / z) / R^{!}(z)-R(-z) / z=\frac{\left(1-2 z-z^{2}\right)\left(1-z-z^{2}+z^{3}-z^{4}\right)}{(1+z)^{3}(1-z)}
$$

We now apply DGAlgebras and we obtain that $H K R$ has five generators $X_{1}, X_{2}, X_{3}, X_{4}, X_{5}$ of degree $(1,2)$, three generators $X_{6}, X_{7}, X_{8}$ of degree $(2,3)$, one generator $X_{9}$ of degree $(2,4)$, three generators $X_{10}, X_{11}, X_{12}$ of degree $(3,5)$, three generators $X_{13}, X_{14}, X_{15}$ of degree $(4,6)$ and one generator $X_{16}$ of degree $(5,7)$. Furthermore these generators satisfy 107 quadratic relations of which only the following four are non-monomial relations:

$$
X_{1} X_{3}+X_{3} X_{4}, X_{1} X_{2}+X_{3} X_{4}, X_{1} X_{8}-X_{4} X_{8}, X_{2} X_{6}-X_{3} X_{6}
$$


and since the following 16 quadratic monomials do not occur among the 107 quadratic relations:

$$
\begin{gathered}
X_{1} X_{4}, X_{1} X_{5}, X_{2} X_{3}, X_{2} X_{4}, X_{2} X_{5}, X_{3} X_{5}, X_{1} X_{7}, X_{2} X_{7}, X_{2} X_{8}, X_{3} X_{7}, \\
X_{4} X_{6}, X_{5} X_{6}, X_{5} X_{8}, X_{6} X_{7}, X_{6} X_{8}, X_{7} X_{8}
\end{gathered}
$$

it follows that $H K R^{!}$is the quotient of the free algebra on the 16 generators $Y_{i}$ (which are dual to the $X_{i}$ ) by the two-sided ideal generated by the 19 quadratic relations

$$
\left[Y_{1}, Y_{4}\right],\left[Y_{1}, Y_{5}\right],\left[Y_{2}, Y_{3}\right],\left[Y_{2}, Y_{4}\right],\left[Y_{2}, Y_{5}\right],\left[Y_{3}, Y_{5}\right],\left[Y_{1}, Y_{7}\right],\left[Y_{2}, Y_{7}\right],\left[Y_{2}, Y_{8}\right],\left[Y_{3}, Y_{7}\right]
$$

$$
\begin{gathered}
{\left[Y_{4}, Y_{6}\right],\left[Y_{5}, Y_{6}\right],\left[Y_{5}, Y_{8}\right],\left[Y_{6}, Y_{7}\right],\left[Y_{6}, Y_{8}\right],\left[Y_{7}, Y_{8}\right],\left[Y_{3}, Y_{4}\right]-\left[Y_{1}, Y_{3}\right]-\left[Y_{1}, Y_{2}\right]} \\
{\left[Y_{1}, Y_{8}\right]+\left[Y_{4}, Y_{8}\right],\left[Y_{2}, Y_{6}\right]+\left[Y_{3}, Y_{6}\right]}
\end{gathered}
$$

Here [,] means the graded commutator so that $\left[Y_{i}, Y_{j}\right]=Y_{i} Y_{j}-Y_{j} Y_{i}$ if $1 \leq i \leq 5$ or $i=10,11,12,16$ and $1 \leq j \leq 5$ or $j=10,11,12,16$. Furthermore we have $\left[Y_{s}, Y_{t}\right]=Y_{s} Y_{t}+Y_{t} Y_{s}$ otherwise. To calculate $H K R^{!}(z)$ we use again the Ufnarovski trick and replace the $Y_{i}$ with $Z_{i} T^{\operatorname{deg} Y_{i}-1}$ everywhere in order to get a new algebra $A$ with generators of degree 1. Calculations in BERGMAN gives after a few minutes

$$
\frac{1}{A(z)}=1-17 z+7 z^{4}+9 z^{5}+2 z^{6}-3 z^{7}-4 z^{8}-4 z^{9}-4 z^{10}-4 z^{11}-4 z^{12}-4 z^{13}-4 z^{14}+O\left(z^{15}\right)
$$

leading as before to

$$
\frac{1}{H K R^{!}(z)}=\frac{1}{A(z)}+17 z-5 z^{2}-4 z^{3}-3 z^{4}-3 z^{5}-z^{6}=\frac{(1+z)^{2}\left(1-3 z+4 z^{3}-2 z^{5}-z^{6}\right)}{1-z}
$$

Furthermore the cube of the augmentation ideal of $H K R$ is equal to zero, and the square of that ideal is generated by

$$
\begin{gathered}
X_{3} X_{5}, X_{2} X_{5}, X_{1} X_{5}, X_{3} X_{4}, X_{2} X_{4}, X_{1} X_{4}, X_{2} X_{3}, X_{5} X_{8}, X_{4} X_{8}, X_{2} X_{8}, X_{3} X_{7}, X_{2} X_{7}, X_{1} X_{4}, \\
X_{5} X_{6}, X_{4} X_{6}, X_{3} X_{6}, X_{7} X_{8}, X_{6} X_{8}, X_{6} X_{7}
\end{gathered}
$$

leading to the bigraded Hilbert series

$$
H K R(x, y)=1+5 x y+4 x y^{2}+3 x y^{3}+3 x y^{4}+x y^{5}+7 x^{2} y^{2}+9 x^{2} y^{3}+3 x^{2} y^{4}
$$

and thus $H(-z, z)=1-5 z^{2}-4 z^{3}+4 z^{4}+6 z^{5}+2 z^{6}$ so that

$$
\frac{1}{P_{H K R}(z)}=(1+1 / z) / H K R^{!}(z)-\left(1-5 z^{2}-4 z^{3}+4 z^{4}+6 z^{5}+2 z^{6}\right) / z
$$

so that

$$
P_{H K R}(z)=\frac{1-z}{(1+z)^{2}\left(1-2 z-z^{2}\right)\left(1-z-z^{2}+z^{3}+z^{4}\right)}
$$

But

$$
P_{R}(z)=\frac{(1+z)^{3}(1-z)}{\left(1-2 z-z^{2}\right)\left(1-z-z^{2}+z^{3}+z^{4}\right)}
$$

so the Avramov spectral sequence degenerates. Furthermore $\operatorname{Ext}_{H K R}^{*}(k, k)$ is not finitely generated.

More details: Case III Now the ring is $R=k[x, y, z, u, v] /\left(x z+y u, z u+u v, y v, z^{2}, v^{2}\right)$. We have that

$$
R^{!}(z)=\left(\frac{1+z}{1-z-z^{2}}\right)^{2} \prod_{n=1}^{\infty} \frac{1+z^{2 n-1}}{1-z^{2 n}}
$$


and

$$
R(z)=\frac{1+3 z+z^{2}-2 z^{3}+z^{4}}{(1-z)^{2}}
$$

and the formula (it is still valid)

$$
\frac{1}{P_{R}(z)}=(1+1 / z) / R^{!}(z)-R(-z) / z
$$

gives $P_{R}(z)$.

On the other hand DGAlgebras gives that $H K R$ has five generators $X_{1}, X_{2}, X_{3}, X_{4}, X_{5}$ of degree $(1,2)$, three generators $X_{5}, X_{7}, X_{8}$ of degree $(2,3)$, one generator $X_{9}$ of degree $(4,6)$ and one generator $X_{10}$ of degree $(5,7)$. Furthermore, these generators satisfy 29 quadratic relations of which only the following are non-monomial relations:

$$
X_{1} X_{3}+X_{3} X_{4}, X_{1} X_{2}+X_{3} X_{5}, X_{1} X_{8}-X_{4} X_{8}, X_{1} X_{6}+X_{3} X_{7}+X_{5} X_{8}
$$

Since $I H K R^{3}=0$ and

$$
\begin{gathered}
\text { IHKR R }=\left(X_{3} X_{5}, X_{2} X_{5}, X_{1} X_{5}, X_{3} X_{4}, X_{2} X_{4}, X_{1} X_{4}, X_{2} X_{3}, X_{5} X_{8}, X_{4} X_{8}, X_{2} X_{8}, X_{3} X_{7},\right. \\
\left.X_{2} X_{7}, X_{1} X_{7}, X_{5} X_{6}, X_{4} X_{6}, X_{3} X_{6}, X_{2} X_{6}, X_{7} X_{8}, X_{6} X_{8}, X_{6} X_{7}, X_{6} X_{6}\right)
\end{gathered}
$$

It follows that $H K R^{!}$is the quotient of the free algebra on the ten generators $Y_{i}$ (which are dual to the $X_{i}$ ) by the twosided ideal generated by the 21 quadratic relations

$$
\begin{gathered}
{\left[Y_{1}, Y_{4}\right],\left[Y_{1}, Y_{5}\right],\left[Y_{2}, Y_{3}\right],\left[Y_{2}, Y_{4}\right],\left[Y_{2}, Y_{5}\right],\left[Y_{1}, Y_{2}\right]-\left[Y_{3}, Y_{5}\right],\left[Y_{1}, Y_{3}\right]-\left[Y_{3}, Y_{4}\right],} \\
{\left[Y_{1}, Y_{7}\right],\left[Y_{2}, Y_{6}\right],\left[Y_{2}, Y_{7}\right],\left[Y_{2}, Y_{8}\right],\left[Y_{3}, Y_{6}\right],\left[Y_{4}, Y_{6}\right],\left[Y_{5}, Y_{6}\right],\left[Y_{1}, Y_{6}\right]-\left[Y_{3}, Y_{7}\right],} \\
{\left[Y_{3}, Y_{7}\right]-\left[Y_{5}, Y_{8}\right],\left[Y_{1}, Y_{8}\right]-\left[Y_{5}, Y_{8}\right], Y_{6} Y_{6},\left[Y_{6}, Y_{7}\right],\left[Y_{6}, Y_{8}\right],\left[Y_{7}, Y_{8}\right]}
\end{gathered}
$$

where [,] again means the graded commutator so that $\left[Y_{i}, Y_{j}\right]=Y_{i} Y_{j}-Y_{j} Y_{i}$ if $1 \leq i \leq 5$ and $1 \leq j \leq 5$, Furthermore $\left[Y_{i}, Y_{j}\right]=Y_{i} Y_{j}+Y_{j} Y_{i}$ otherwise. Note that here the generators should have the degree 2,3,4,5 and we again apply the Ufnarovski trick introducing a new extra variable $\mathrm{T}$ and replacing everywhere $Y_{i}$ by $T^{\operatorname{deg} Y_{i}-1} Z_{i}$ gives a new algebra $A$ with generators of degree 1 , such that

$$
1 / H K R^{!}(z)=1 / A(z)+11 z-5 z^{2}-3 z^{3}-x^{5}-z^{6}
$$

Again BERGMAN give $A(z)$ easily up to degree 20 and we get:

$$
\begin{gathered}
1 / H K R^{!}(z)=1-5 z^{2}-3 z^{3}+7 z^{4}+9 z^{5}+3 z^{6}-5 z^{7}-13 z^{8}-11 z^{9}+2 z^{10}+9 z^{11}+z^{12}-5 z^{13}+ \\
+2 z^{14}+7 z^{15}+4 z^{16}+5 z^{17}+6 z^{18}-2 * z^{19}-8 z^{20} \ldots
\end{gathered}
$$

and since $\operatorname{HKR}(x, y)=1+5 x y+3 x y^{2}+x y^{4}+x y^{5}+7 x^{2} y^{2}+10 x^{2} y^{3}+4 x^{2} y^{4}$ we get $H K R(-z, z)=$ $1-5 z^{2}-3 z^{3}+7 z^{4}+9 z^{5}+3 z^{5}$ and the Löfwall formula

$$
1 / \Phi_{R}(z, z)=1 / P_{H K R}(z, z)=(1+1 / z) / H K R^{!}(z)-H K R(-z, z) / z
$$

gives

$$
\Phi_{R}(z, z)=1+5 z^{2}+3 z^{3}+18 z^{4}+21 z^{5}+66 z^{6}+111 z^{7}+274 z^{8}+549 z^{9}+1251 * z^{10} \ldots
$$

and the Avramov spectral sequence degenerates up to degree 20 (and probably up to degree $\infty$ ).

\section{References}

[A] D.J. Anick, A counterexample to a conjecture of Serre, Ann. of Math. 115, 1982, pp. 1-33; Correction, Ann. of Math. 116 , p. 661. 
[A-G] Avramov, L. L.; Golod, E. S., The homology of algebra of the Koszul complex of a local Gorenstein ring. (Russian), Mat. Zametki 9, (1971), pp. 53-58. English translation [Math. Notes 9 (1971), 30-32].

[AV1] Avramov, L. L.,Obstructions to the existence of multiplicative structures on minimal free resolutions. Amer. J. Math., 103 (1981), pp. 1-31.

[AV2] Avramov, L. L., Infinite free resolutions, in Six lectures on commutative algebra (Bellaterra, 1996), Progr. Math., 166, Birkhuser, Basel, 1998, pp. 1-118.

[BA] Backelin, J. et al, BERGMAN, A programme for (non-commutative) Gröbner basis calculations available at /http://servus.math.su.se/bergman/

[Ei-K] D. Eisenbud, J. Koh, Nets of alternating matrices and the linear syzygy conjectures, Adv. Math.106 (1994), pp. 1-35.

[GOV] Govorov, V. E. Graded algebras. (Russian) Mat. Zametki 12, (1972), pp. 197-204. English translation [Math. Notes 12 (1973), 556-562].

[KAT] L. Katthän, A non-Golod ring with a trivial product on its Koszul homology, http://arxiv.org/pdf/1511.04883.pdf

[LE] J.-M. Lemaire, Algèbres Connexes et Homologie des Espaces de Lacets, Lecture Notes in Mathematics, vol. 422, Springer-Verlag, 1974.

[L1] Löfwall, C., On the subalgebra generated by one-dimensional elements in the Yoneda Ext-algebra, in Algebra, algebraic topology, and their interactions, ( J.-E. Roos, ed), Lecture Notes in Math., vol.1183, Springer-Verlag, Berlin-New York, 1986, pp. 291-338.

[L2] C. Löfwall, The Yoneda Ext-algebra for an equi-characteristic local ring $(R, m)$ with $m^{3}=0$, unpublished manuscript, circa 1976.

[LR1] C. Löfwall and J.-E. Roos, Cohomologie des algèbres de Lie graduées et séries de Poincaré-Betti non rationnelles, C.R. Acad. Sc. Paris 290, 1980, pp. A733-A736.

[LR2] C. Löfwall and J.-E. Roos, A nonnilpotent 1-2-presented graded Hopf algebra whose Hilbert series converges in the unit circle, Adv. Math.130, 1997, pp. 161-200.

[MOO] Moore, Frank, DGAlgebras. A package for Macaulay2 that is used to define and manipulate DG algebras, available at http://www.math.uiuc.edu/Macaulay2/Packages/

[R1] J.-E. Roos, Relations between the Poincaré-Betti series of Loop Spaces and of Local rings, Springer Lecture Notes in Math.740, 1979, 285-322.

[R2] J.-E. Roos, On the use of graded Lie algebras in the theory of local rings, Commutative algebra: Durham 1981 (R. Y. Sharp, ed.) London Math. Soc. Lecture Notes Ser. vol. 72, Cambridge Univ. Press, Cambridge, 1982, pp. 204230.

[R3] J.-E. Roos, A computer-aided study of the graded Lie-algebra of a local commutative noetherian ring (with an Appendix by Clas Löfwall), Journal of Pure and Applied Algebra 91 , 1994, pp. 255-315.

[U] Ufnarovskij, V., Combinatorial and Asymptotic Methods in Algebra, in Encyclopaedia of Mathematical Sciences, vol. 57, Algebra VI

(A.I. Kostrikin and I.R. Shafarevich, eds.), Springer, Berlin, 1994, pp. 1-196. 


\title{
APPENDIX 1 \\ A Description of the Homological Behaviour of families of quadratic forms in four variables.
}

\author{
Jan-Erik Roos \\ Department of Mathematics \\ University of Stockholm \\ S-106 91 Stockholm, SWEDEN \\ email: jeroos@math.su.se
}

This is Appendix 1 to our present paper (2015) about the homological properties of the homology of the Koszul algebra of codim $\geq 4$ local rings. Part of this appendix was originally included as pages 86-95 in the volume "Syzygies and Geometry", october 7-8, 1995, AMS Special Session and International Conference, Northeastern University, Boston 1995, edited by Antony Iarrobino, Alex Martsinkovsky and Jerzy Weyman. But that volume had limited circulation, and here we present an update where furthermore hopefully all misprints in the tables have been corrected (I thank Aldo Conca for help with this).

Let $k$ be a field (for simplicity we assume here that it is of characteristic 0 ), $k\left[X_{1}, \ldots, X_{n}\right]$ the commutative polynomial ring in $n$ variables over $k$ and let $f_{1}, \ldots, f_{t}$ be quadratic forms in the $X_{i}$ :s. Consider the quotient ring

$$
R=k\left[X_{1}, \ldots, X_{n}\right] /\left(f_{1}, \ldots f_{r}\right)
$$

which is a graded vector space over $k$. If $V$ is a vector space over $k$, we denote by $|V|$ the $\operatorname{dimension} \operatorname{dim}_{k}(V)$ of $V$ over $k$. Let $R(t)=\sum_{i=0}^{\infty}\left|R^{i}\right| t^{i}$ be the Hilbert series of $R$. In Tables 1-2 below you find a description of the 60 possible series $R(t)$ when $n=4$ (the "example" column in Tables 1-2 refers to the examples in Tables 3-7 below).

Let $\operatorname{Ext}_{R}^{*}(k, k)=\operatorname{Ext}_{R}^{*, *}(k, k)$ be the Yoneda Ext-algebra of $R$. This space is dual to $\operatorname{Tor}_{*, *}^{R}(k, k)$ and the second grading comes from the grading of $R$. In Tables 3-7 you find the possible 83 series

$$
P_{R}(x, y)=\sum_{i, j \geq 0}\left|\operatorname{Tor}_{i, j}^{R}(k, k)\right| x^{i} y^{j}
$$

for $n=4$. Indeed, these Tables 3-7 should be read in conjunction with Tables 1-2 and Table 8 which describes the relevant non Koszul cases. Let me be more precise: The sub-algebra of $\operatorname{Ext}_{R}^{*}(k, k)$ generated by $\operatorname{Ext}_{R}^{1}(k, k)$ is denoted by $R^{!}$and its Hilbert series by $R^{!}(t)$. For so-called Koszul algebras $R$ we have $P_{R}(x, y)=R^{!}(x y)$ which implies in particular (put $x=-1, y=t$ ) that

$$
R(t) R^{!}(-t)=1 \text {. }
$$

In all cases for $n \leq 4$, except one case, we have the formula

$$
P_{R}(x, y)^{-1}=(1+1 / x) / R^{!}(x y)-R(-x y) / x,
$$

and in the only exceptional case (case $\mathbf{1 2}$ of Table 3) we have

$$
P_{R}(x, y)^{-1}=\left(1-1 / x^{2}\right) / R^{!}(x y)+R(-x y) / x^{2} .
$$

The Tables 3-7 give the Betti numbers as the programme MACAULAY presents them; thus the first horizontal line for each case gives the $\left|\operatorname{Tor}_{i, i}^{R}(k, k)\right|$, the second line gives the $\left|\operatorname{Tor}_{i, i+1}^{R}(k, k)\right|$, etc. If the ring is a Koszul algebra there is only one horizontal line and if $R(t)$ is given as e.g. $H_{2}$ (which happens in case $\mathbf{8 1}$ ), then the corresponding $R^{!}(t)$ (which is determined by (3)) is denoted by e.g. $A_{2}$. In the non Koszul cases 
we have special notations for the $R^{!}(t)$ which are given explicitly in Table 8, and we use the formula (4) and (in case 12) the formula (5) to get $P_{R}(x, y)$.

Among these 83 cases there are 68 depth 0 cases which are new (they are given in boldface in Tables 2-7) and 15 cases of positive depth which correspond to $n \leq 3$ and which were known before [1].

Furthermore $\operatorname{Ext}_{R}^{*, *}(k, k)$ and its subalgebra $R^{!}$are not only Hopf algebras, but also enveloping algebras of graded Lie algebras $g^{*, *}$ and $\eta^{*}$. In [3,4] the relations between the $g^{*, *}$ and $\eta^{*}$ are explained. The Lie algebras $\eta^{*}$ that correspond to Table 8 are either nilpotent (maximal degree of nilpotency is 4 , which means that $\eta^{5}=0$ but $\eta^{4} \neq 0$ ) or nice extensions of free Lie algebras (or product of two free Lie algebras) by nilpotent Lie algebras.

Remarks about the proofs: In all the 83 cases the corresponding dual algebra $R^{!}$can be written down and it is a quotient of a free algebra in 4 variables with an ideal generated by quadratic elements. We now want to determine the Hilbert series of that quotient, i.e. $R^{!}(t)$. For this we use the programme BERGMAN which calculates a Gröbner basis of that ideal. It turns out that in all the 83 cases we can find a suitable permutation of the variables for which this ideal has a finite Gröbner basis. Then it follows that the quotient has a rational series (Govorov and Backelin). Let me illustrate this explicitly for the most complicated case in table 8, namely case 37 . Here $R_{37}=k[x, y, z, u] /\left(x^{2}, y^{2}, x y-z u, y z-x u,(x-y)(z-u)\right)$. It follows that the Koszul dual $R_{37}^{!}$is given by the formula:

$$
R_{37}^{!}=\frac{k<X, Y, Z, U>}{\left(Z^{2}, U^{2}, X Y+Y X+Z U+U Z, X Z+Z X-Y U-U Y, 2 X Z+2 Z X+Y Z+Z Y+X U+U X\right)}
$$

where $k\langle X, Y, Z, U\rangle$ is the free associative algebra in the dual variables $X, Y, Z, U$. When we plug this into BERGMAN we see that the correspondig ideal has an infinite Gröbner basis. However, using the permutebergman addition by Backelin to BERGMAN as described in [5] we see that the order of the variables $(\mathrm{X}, \mathrm{U}, \mathrm{Z}, \mathrm{Y})$ gives a finite Gröbner basis with 5 elements in degree 2, 2 elements in degree 3, 2 in degree 4, 1 in degree 5 and 1 in degree 6 . Therefore the Hilbert series is rational and can be explicitly determined. Furthermore it is easy to see that the conditions $L_{3}$ are satisfied in all cases, except case 12 of Table 3 , where we have $L_{4}$. This explains the formulae (4) and (5). Our results are also related to the rational homotopy Lie algebras of CW-complexes [2],[6].

\section{BIBLOGRAPHY}

[1] Backelin,J. and Fröberg,R., Poincaré series of short artinian rings, J. of Algebra, 96, 1985, pp. 495-498.

[2] J.-M. Lemaire, Algèbres Connexes et Homologie des Espaces de Lacets, Lecture Notes in Mathematics, vol. 422, Springer-Verlag, 1974.

[3] Löfwall,C. On the subalgebra generated by one-dimensional elements in the Yoneda Ext-algebra Algebra, algebraic topology, and their interactions, ( J.-E. Roos, ed), Lecture Notes in Math., vol. 1183, Springer-Verlag, Berlin-New York, 1986, pp. 291-338.

[4] J.-E. Roos, A computer-aided study of the graded Lie-algebra of a local commutative noetherian ring (with an Appendix by Clas Löfwall), Journal of Pure and Applied Algebra 91,1994 pp. 255-315.

[5] J.-E. Roos, Three-dimensional manifolds, skew-Gorenstein rings and their cohomology. J. Commut. Algebra 2 (2010), no. 4, pp 473-499.

[6] J.-E. Roos, Relations between the Poincaré-Betti series of Loop Spaces and of Local rings, Springer Lecture Notes in Math.740, 1979, 285-322. 
Table 1. THE FIRST 30 POSSIBLE HILBERT SERIES $H_{1}, \ldots, H_{30}$

\begin{tabular}{|c|c|c|c|c|c|c|c|c|c|}
\hline & Rational form & \multicolumn{7}{|c|}{ Taylor series (Hilbert function) } & Example \\
\hline$H_{1}$ & $1+4 t$ & 1 & 4 & 0 & 0 & 0 & 0 & 0 & 83 \\
\hline$H_{2}$ & $1+4 t+t^{2}$ & 1 & 4 & 1 & 0 & 0 & 0 & 0 & 81 \\
\hline$H_{3}$ & $\left(1+3 t-3 t^{2}\right) /(1-t)$ & 1 & 4 & 1 & 1 & 1 & 1 & 1 & 82 \\
\hline$H_{4}$ & $1+4 t+2 t^{2}$ & 1 & 4 & 2 & 0 & 0 & 0 & 0 & 78 \\
\hline$H_{5}$ & $\left(1+3 t-2 t^{2}-t^{3}\right) /(1-t)$ & 1 & 4 & 2 & 1 & 1 & 1 & 1 & 79 \\
\hline$H_{6}$ & $\left(1+3 t-2 t^{2}\right) /(1-t)$ & 1 & 4 & 2 & 2 & 2 & 2 & 2 & 80 \\
\hline$H_{7}$ & $1+4 t+3 t^{2}=(1+t)(1+3 t)$ & 1 & 4 & 3 & 0 & 0 & 0 & 0 & 71 \\
\hline$H_{8}$ & $1+4 t+3 t^{2}+t^{3}$ & 1 & 4 & 3 & 1 & 0 & 0 & 0 & 73 \\
\hline$H_{9}$ & $\left(1+3 t-t^{2}-2 t^{3}\right) /(1-t)$ & 1 & 4 & 3 & 1 & 1 & 1 & 1 & 72,74 \\
\hline$H_{10}$ & $\left(1+3 t-t^{2}-t^{3}\right) /(1-t)$ & 1 & 4 & 3 & 2 & 2 & 2 & 2 & 75 \\
\hline$H_{11}$ & $\left(1+3 t-t^{2}\right) /(1-t)$ & 1 & 4 & 3 & 3 & 3 & 3 & 3 & 76 \\
\hline$H_{12}$ & $\left(1+2 t-4 t^{2}+2 t^{3}\right) /(1-t)^{2}$ & 1 & 4 & 3 & 4 & 5 & 6 & 7 & 77 \\
\hline$H_{13}$ & $1+4 t+4 t^{2}=(1+2 t)^{2}$ & 1 & 4 & 4 & 0 & 0 & 0 & 0 & $54,55,56,57$ \\
\hline$H_{14}$ & $1+4 t+4 t^{2}+t^{3}=(1+t)\left(1+3 t+t^{2}\right)$ & 1 & 4 & 4 & 1 & 0 & 0 & 0 & 61 \\
\hline$H_{15}$ & $\left(1+3 t-3 t^{3}\right) /(1-t)$ & 1 & 4 & 4 & 1 & 1 & 1 & 1 & $58,59,60,62$ \\
\hline$H_{16}$ & $\left(1+3 t-2 t^{3}-t^{4}\right) /(1-t)$ & 1 & 4 & 4 & 2 & 1 & 1 & 1 & 64 \\
\hline$H_{17}$ & $\left(1+2 t-2 t^{2}\right)(1+t) /(1-t)$ & 1 & 4 & 4 & 2 & 2 & 2 & 2 & 63,65 \\
\hline$H_{18}$ & $\left(1+3 t-t^{3}\right) /(1-t)$ & 1 & 4 & 4 & 3 & 3 & 3 & 3 & 66 \\
\hline$H_{19}$ & $(1+3 t) /(1-t)$ & 1 & 4 & 4 & 4 & 4 & 4 & 4 & 68 \\
\hline$H_{20}$ & $\left(1+2 t-3 t^{2}+t^{4}\right) /(1-t)^{2}$ & 1 & 4 & 4 & 4 & 5 & 6 & 7 & 67,69 \\
\hline$H_{21}$ & $\left(1+2 t-3 t^{2}+t^{3}\right) /(1-t)^{2}$ & 1 & 4 & 4 & 5 & 6 & 7 & 8 & 70 \\
\hline$H_{22}$ & $1+4 t+5 t^{2}$ & 1 & 4 & 5 & 0 & 0 & 0 & 0 & 29 \\
\hline$H_{23}$ & $1+4 t+5 t^{2}+t^{3}$ & 1 & 4 & 5 & 1 & 0 & 0 & 0 & 30,32 \\
\hline$H_{24}$ & $\left(1+3 t+t^{2}-4 t^{3}\right) /(1-t)$ & 1 & 4 & 5 & 1 & 1 & 1 & 1 & 31,33 \\
\hline$H_{25}$ & $1+4 t+5 t^{2}+2 t^{3}=(1+t)^{2}(1+2 t)$ & 1 & 4 & 5 & 2 & 0 & 0 & 0 & 38 \\
\hline$H_{26}$ & $\left(1+3 t+t^{2}-3 t^{3}-t^{4}\right) /(1-t)$ & 1 & 4 & 5 & 2 & 1 & 1 & 1 & 39 \\
\hline$H_{27}$ & $\left(1+3 t+t^{2}-3 t^{3}\right) /(1-t)$ & 1 & 4 & 5 & 2 & 2 & 2 & 2 & $34,35,36,37,40$ \\
\hline$H_{28}$ & $\left(1+2 t-t^{2}-t^{3}\right)(1+t) /(1-t)$ & 1 & 4 & 5 & 3 & 2 & 2 & 2 & 43 \\
\hline$H_{29}$ & $(1+2 t)\left(1+t-t^{2}\right) /(1-t)$ & 1 & 4 & 5 & 3 & 3 & 3 & 3 & $41,42,44$ \\
\hline$H_{30}$ & $\left(1+2 t-t^{2}\right)(1+t) /(1-t)$ & 1 & 4 & 5 & 4 & 4 & 4 & 4 & 46 \\
\hline
\end{tabular}

On the next page you can see the last 30 possible Hilbert series. 
Table 2. THE LAST 30 POSSIBLE HILBERT SERIES $H_{31}, \ldots, H_{60}$

\begin{tabular}{|c|c|c|c|c|c|c|c|c|c|}
\hline & Rational factored form & \multicolumn{7}{|c|}{ Taylor series (Hilbert function) } & Example \\
\hline$H_{31}$ & $\left(1+2 t-2 t^{2}-2 t^{3}+2 t^{4}\right) /(1-t)^{2}$ & 1 & 4 & 5 & 4 & 5 & 6 & 7 & $45,47,48$ \\
\hline$H_{32}$ & $\left(1+3 t+t^{2}\right) /(1-t)$ & 1 & 4 & 5 & 5 & 5 & 5 & 5 & 50 \\
\hline$H_{33}$ & $\left(1+2 t-2 t^{2}-t^{3}+t^{4}\right) /(1-t)^{2}$ & 1 & 4 & 5 & 5 & 6 & 7 & 8 & 49,51 \\
\hline$H_{34}$ & $\left(1+2 t-2 t^{2}\right) /(1-t)^{2}$ & 1 & 4 & 5 & 6 & 7 & 8 & 9 & 52 \\
\hline$H_{35}$ & $\left(1+2 t-2 t^{2}+t^{3}\right) /(1-t)^{2}$ & 1 & 4 & 5 & 7 & 9 & 11 & 13 & 53 \\
\hline$H_{36}$ & $1+4 t+6 t^{2}+4 t^{3}+t^{4}$ & 1 & 4 & 6 & 4 & 1 & 0 & 0 & 11 \\
\hline$H_{37}$ & $\left(1+3 t+2 t^{2}-2 t^{3}-3 t^{4}\right) /(1-t)$ & 1 & 4 & 6 & 4 & 1 & 1 & 1 & 12 \\
\hline$H_{38}$ & $\left(1+3 t+2 t^{2}-2 t^{3}-2 t^{4}\right) /(1-t)$ & 1 & 4 & 6 & 4 & 2 & 2 & 2 & 13 \\
\hline$H_{39}$ & $\left(1+3 t+2 t^{2}-2 t^{3}-t^{4}\right) /(1-t)$ & 1 & 4 & 6 & 4 & 3 & 3 & 3 & 14 \\
\hline$H_{40}$ & $\left(1+3 t+2 t^{2}-2 t^{3}\right) /(1-t)$ & 1 & 4 & 6 & 4 & 4 & 4 & 4 & 15 \\
\hline$H_{41}$ & $\left(1+2 t-t^{2}-4 t^{3}+3 t^{4}\right) /(1-t)^{2}$ & 1 & 4 & 6 & 4 & 5 & 6 & 7 & 16 \\
\hline$H_{42}$ & $\left(1+t-t^{2}\right)(1+t)^{2} /(1-t)$ & 1 & 4 & 6 & 5 & 4 & 4 & 4 & 18 \\
\hline$H_{43}$ & $\left(1+3 t+2 t^{2}-t^{3}\right) /(1-t)$ & 1 & 4 & 6 & 5 & 5 & 5 & 5 & 17,19 \\
\hline$H_{44}$ & $\left(1+2 t-t^{2}-3 t^{3}+2 t^{4}\right) /(1-t)^{2}$ & 1 & 4 & 6 & 5 & 6 & 7 & 8 & 20 \\
\hline$H_{45}$ & $(1+t)(1+2 t) /(1-t)$ & 1 & 4 & 6 & 6 & 6 & 6 & 6 & 23 \\
\hline$H_{46}$ & $\left(1+t-t^{2}\right)^{2} /(1-t)^{2}$ & 1 & 4 & 6 & 6 & 7 & 8 & 9 & $21,22,24$ \\
\hline$H_{47}$ & $\left(1+2 t-t^{2}-t^{3}\right) /(1-t)^{2}$ & 1 & 4 & 6 & 7 & 8 & 9 & 10 & 26 \\
\hline$H_{48}$ & $\left(1+t-2 t^{2}+t^{3}\right)(1+t) /(1-t)^{2}$ & 1 & 4 & 6 & 7 & 9 & 11 & 13 & 25 \\
\hline$H_{49}$ & $\left(1+2 t-t^{2}\right) /(1-t)^{2}$ & 1 & 4 & 6 & 8 & 10 & 12 & 14 & 27 \\
\hline$H_{50}$ & $\left(1+t-3 t^{2}+3 t^{3}-t^{4}\right) /(1-t)^{3}$ & 1 & 4 & 6 & 10 & 15 & 21 & 28 & 28 \\
\hline$H_{51}$ & $(1+t)^{3} /(1-t)$ & 1 & 4 & 7 & 8 & 8 & 8 & 8 & 5 \\
\hline$H_{52}$ & $\left(1+2 t-2 t^{3}\right) /(1-t)^{2}$ & 1 & 4 & 7 & 8 & 9 & 10 & 11 & 6 \\
\hline$H_{53}$ & $\left(1+2 t-2 t^{3}+t^{4}\right) /(1-t)^{2}$ & 1 & 4 & 7 & 8 & 10 & 12 & 14 & 7 \\
\hline$H_{54}$ & $\left(1+t-t^{2}\right)(1+t) /(1-t)^{2}$ & 1 & 4 & 7 & 9 & 11 & 13 & 15 & 8 \\
\hline$H_{55}$ & $(1+2 t) /(1-t)^{2}$ & 1 & 4 & 7 & 10 & 13 & 16 & 19 & 9 \\
\hline$H_{56}$ & $\left(1+t-2 t^{2}+t^{3}\right) /(1-t)^{3}$ & 1 & 4 & 7 & 11 & 16 & 22 & 29 & 10 \\
\hline$H_{57}$ & $(1+t)^{2} /(1-t)^{2}$ & 1 & 4 & 8 & 12 & 16 & 20 & 24 & 3 \\
\hline$H_{58}$ & $\left(1+t-t^{2}\right) /(1-t)^{3}$ & 1 & 4 & 8 & 13 & 19 & 26 & 34 & 4 \\
\hline$H_{59}$ & $(1+t) /(1-t)^{3}$ & 1 & 4 & 9 & 16 & 25 & 36 & 49 & 2 \\
\hline$H_{60}$ & $1 /(1-t)^{4}$ & 1 & 4 & 10 & 20 & 35 & 56 & 84 & 1 \\
\hline
\end{tabular}


Table 3. The first 19 cases (case numbers in boldface indicate depth 0 rings).

\begin{tabular}{|c|c|c|c|c|c|c|c|c|c|c|c|}
\hline Case & \multicolumn{8}{|c|}{ The graded Betti Numbers } & ideal gen:s (ex.) & $R(t)$ & $R^{!}(t)$ \\
\hline 1 & 1 & 4 & 6 & 4 & 1 & 0 & 0 & 0 & 0 & $H_{60}$ & $A_{60}$ \\
\hline 2 & 1 & 4 & 7 & 8 & 8 & 8 & 8 & 8 & $x^{2}$ & $H_{59}$ & $A_{59}$ \\
\hline 3 & 1 & 4 & 8 & 12 & 16 & 20 & 24 & 28 & $x^{2}, y^{2}$ & $H_{57}$ & $A_{57}$ \\
\hline 4 & 1 & 4 & 8 & 13 & 21 & 34 & 55 & 89 & $x^{2}, x y$ & $H_{58}$ & $A_{58}$ \\
\hline 5 & 1 & 4 & 9 & 16 & 25 & 36 & 49 & 64 & $x^{2}, y^{2}, z^{2}$ & $H_{51}$ & $A_{51}$ \\
\hline 6 & $\begin{array}{l}1 \\
- \\
-\end{array}$ & $\begin{array}{l}4 \\
- \\
-\end{array}$ & $\begin{array}{l}9 \\
- \\
-\end{array}$ & $\begin{array}{r}16 \\
1 \\
-\end{array}$ & $\begin{array}{r}25 \\
6 \\
-\end{array}$ & $\begin{array}{r}36 \\
21 \\
-\end{array}$ & $\begin{array}{r}49 \\
56 \\
1\end{array}$ & $\begin{array}{r}64 \\
126 \\
8\end{array}$ & $x^{2}, y^{2}+x z, y z$ & $H_{52}$ & $A_{51}$ \\
\hline 7 & $\begin{array}{l}1 \\
- \\
-\end{array}$ & $\begin{array}{l}4 \\
- \\
-\end{array}$ & $\begin{array}{l}9 \\
- \\
-\end{array}$ & $\begin{array}{r}16 \\
2 \\
-\end{array}$ & $\begin{array}{r}25 \\
12 \\
-\end{array}$ & $\begin{array}{r}36 \\
42 \\
-\end{array}$ & $\begin{array}{r}49 \\
112 \\
4\end{array}$ & $\begin{array}{r}64 \\
252 \\
32\end{array}$ & $\begin{array}{l}x^{2}+y^{2}, z^{2}+u^{2} \\
x z+y u\end{array}$ & $H_{53}$ & $A_{51}$ \\
\hline 8 & 1 & 4 & 9 & 17 & 30 & 51 & 85 & 140 & $x^{2}, y^{2}, x z$ & $H_{54}$ & $A_{54}$ \\
\hline 9 & 1 & 4 & 9 & 18 & 36 & 72 & 144 & 288 & $x^{2}, x y, y^{2}$ & $H_{55}$ & $A_{55}$ \\
\hline 10 & 1 & 4 & 9 & 19 & 41 & 88 & 189 & 406 & $x^{2}, x y, x z$ & $H_{56}$ & $A_{56}$ \\
\hline 11 & 1 & 4 & 10 & 20 & 35 & 56 & 84 & 120 & $x^{2}, y^{2}, z^{2}, u^{2}$ & $H_{36}$ & $A_{36}$ \\
\hline 12 & $\begin{array}{l}1 \\
- \\
- \\
- \\
-\end{array}$ & $\begin{array}{l}4 \\
- \\
- \\
- \\
-\end{array}$ & $\begin{array}{r}10 \\
- \\
- \\
- \\
-\end{array}$ & $\begin{array}{r}20 \\
- \\
1 \\
- \\
-\end{array}$ & $\begin{array}{r}35 \\
- \\
7 \\
- \\
-\end{array}$ & $\begin{array}{r}56 \\
- \\
29 \\
- \\
-\end{array}$ & $\begin{array}{r}84 \\
- \\
91 \\
- \\
1\end{array}$ & $\begin{array}{r}120 \\
- \\
239 \\
- \\
10\end{array}$ & $\begin{array}{l}x^{2}+x y, y^{2}+x u \\
z^{2}+x u, u^{2}+z u\end{array}$ & $H_{37}$ & $A_{36}$ \\
\hline 13 & $\begin{array}{l}1 \\
- \\
-\end{array}$ & $\begin{array}{l}4 \\
- \\
-\end{array}$ & $\begin{array}{r}10 \\
- \\
-\end{array}$ & $\begin{array}{r}20 \\
1 \\
-\end{array}$ & $\begin{array}{r}35 \\
6 \\
-\end{array}$ & $\begin{array}{r}56 \\
22 \\
-\end{array}$ & $\begin{array}{r}84 \\
62 \\
1\end{array}$ & $\begin{array}{r}120 \\
148 \\
8\end{array}$ & $\begin{array}{l}x^{2}+z^{2}+u^{2} \\
y^{2}, x z, y u+z u\end{array}$ & $H_{38}$ & $A_{36}$ \\
\hline 14 & 1 & $\begin{array}{l}4 \\
- \\
-\end{array}$ & $\begin{array}{r}10 \\
- \\
-\end{array}$ & $\begin{array}{r}20 \\
2 \\
-\end{array}$ & $\begin{array}{r}35 \\
13 \\
-\end{array}$ & $\begin{array}{r}56 \\
51 \\
-\end{array}$ & $\begin{array}{r}84 \\
153 \\
4\end{array}$ & $\begin{array}{r}120 \\
387 \\
36\end{array}$ & $\begin{array}{l}x z, y^{2}, z^{2}+u^{2}, \\
y u+z u\end{array}$ & $H_{39}$ & $A_{36}$ \\
\hline 15 & 1 & $\begin{array}{l}4 \\
- \\
-\end{array}$ & $\begin{array}{r}10 \\
- \\
-\end{array}$ & $\begin{array}{r}20 \\
3 \\
-\end{array}$ & $\begin{array}{r}35 \\
20 \\
-\end{array}$ & $\begin{array}{r}56 \\
80 \\
-\end{array}$ & $\begin{array}{r}84 \\
244 \\
9\end{array}$ & $\begin{array}{r}120 \\
626 \\
84\end{array}$ & $\begin{array}{l}x z, y^{2}, y z+u^{2}, \\
y u+z u\end{array}$ & $H_{40}$ & $A_{36}$ \\
\hline 16 & 1 & $\begin{array}{l}4 \\
- \\
-\end{array}$ & $\begin{array}{r}10 \\
- \\
-\end{array}$ & $\begin{array}{r}20 \\
4 \\
-\end{array}$ & $\begin{array}{r}35 \\
26 \\
-\end{array}$ & $\begin{array}{r}56 \\
103 \\
-\end{array}$ & $\begin{array}{r}84 \\
312 \\
16\end{array}$ & $\begin{array}{l}120 \\
797 \\
144\end{array}$ & $\begin{array}{l}x y+z^{2}+y u, y^{2} \\
y u+z u, x z\end{array}$ & $H_{41}$ & $A_{36}$ \\
\hline 17 & 1 & $\begin{array}{l}4 \\
- \\
\end{array}$ & $\begin{array}{r}10 \\
- \\
\end{array}$ & $\begin{array}{r}21 \\
- \\
\end{array}$ & $\begin{array}{r}39 \\
1 \\
\end{array}$ & $\begin{array}{r}66 \\
8 \\
\end{array}$ & $\begin{array}{r}104 \\
36 \\
\end{array}$ & $\begin{array}{l}155 \\
121 \\
\end{array}$ & $\begin{array}{l}x z, y z+x u \\
y^{2}, y u+z u\end{array}$ & $H_{43}$ & $A_{43.1}$ \\
\hline 18 & 1 & 4 & 10 & 21 & 40 & 72 & 125 & 212 & $x^{2}, y^{2}, z^{2}, y u$ & $H_{42}$ & $A_{42}$ \\
\hline 19 & 1 & $\begin{array}{l}4 \\
- \\
-\end{array}$ & $\begin{array}{r}10 \\
- \\
-\end{array}$ & $\begin{array}{r}21 \\
1 \\
-\end{array}$ & $\begin{array}{r}40 \\
7 \\
-\end{array}$ & $\begin{array}{r}72 \\
29 \\
-\end{array}$ & $\begin{array}{r}125 \\
93 \\
1\end{array}$ & $\begin{array}{r}212 \\
255 \\
10\end{array}$ & $\begin{array}{l}x z, y^{2} \\
y u+z u, u^{2}\end{array}$ & $H_{43}$ & $A_{42}$ \\
\hline
\end{tabular}


Table 4. Cases 20-37

\begin{tabular}{|c|c|c|c|c|c|c|c|c|c|c|c|}
\hline Case & \multicolumn{8}{|c|}{ The graded Betti Numbers } & ideal gen:s (ex.) & $R(t)$ & $R^{!}(t)$ \\
\hline 20 & 1 & $\begin{array}{l}4 \\
- \\
-\end{array}$ & $\begin{array}{r}10 \\
- \\
-\end{array}$ & $\begin{array}{r}21 \\
2 \\
-\end{array}$ & $\begin{array}{r}40 \\
13 \\
-\end{array}$ & $\begin{array}{r}72 \\
52 \\
-\end{array}$ & $\begin{array}{r}125 \\
163 \\
4\end{array}$ & $\begin{array}{r}212 \\
440 \\
36\end{array}$ & $\begin{array}{l}x z, y^{2} \\
y u+z^{2} \\
y u+z u\end{array}$ & $H_{44}$ & $A_{42}$ \\
\hline 21 & 1 & 4 & 10 & 22 & 45 & 88 & 167 & 310 & $x z, y^{2}, z^{2}, y u+z u$ & $H_{46}$ & $A_{46}$ \\
\hline 22 & $\begin{array}{l}1 \\
- \\
\end{array}$ & $\begin{array}{l}4 \\
- \\
\end{array}$ & $\begin{array}{r}10 \\
-\end{array}$ & $\begin{array}{r}22 \\
- \\
\end{array}$ & $\begin{array}{r}45 \\
- \\
\end{array}$ & $\begin{array}{r}88 \\
- \\
\end{array}$ & $\begin{array}{r}167 \\
1\end{array}$ & $\begin{array}{r}311 \\
7\end{array}$ & $\begin{array}{l}x^{2}+x y, x u, \\
x z+y u, y^{2}\end{array}$ & $H_{46}$ & $A_{46.1}$ \\
\hline 23 & 1 & 4 & 10 & 22 & 46 & 94 & 190 & 382 & $x z, x u, y^{2}, z^{2}$ & $H_{45}$ & $A_{45}$ \\
\hline 24 & 1 & $\begin{array}{l}4 \\
- \\
-\end{array}$ & $\begin{array}{r}10 \\
- \\
-\end{array}$ & $\begin{array}{r}22 \\
1 \\
-\end{array}$ & $\begin{array}{r}46 \\
6 \\
-\end{array}$ & $\begin{array}{r}94 \\
23 \\
-\end{array}$ & $\begin{array}{r}190 \\
72 \\
1\end{array}$ & $\begin{array}{r}382 \\
201 \\
8 \\
\end{array}$ & $\begin{array}{l}x z, y^{2} \\
y z+z^{2} \\
y u+z u\end{array}$ & $H_{46}$ & $A_{45}$ \\
\hline 25 & 1 & 4 & 10 & 23 & 51 & 111 & 240 & 517 & $x^{2}, x y, x z, u^{2}$ & $H_{48}$ & $A_{48}$ \\
\hline 26 & 1 & 4 & 10 & 23 & 52 & 117 & 263 & 591 & $x z, y^{2}, y u, z u$ & $H_{47}$ & $A_{47}$ \\
\hline 27 & 1 & 4 & 10 & 24 & 58 & 140 & 338 & 816 & $x y, x z, y^{2}, y z$ & $H_{49}$ & $A_{49}$ \\
\hline 28 & 1 & 4 & 10 & 26 & 69 & 181 & 476 & 1252 & $x^{2}, x y, x z, x u$ & $H_{50}$ & $A_{50}$ \\
\hline 29 & $\begin{array}{l}1 \\
- \\
-\end{array}$ & $\begin{array}{l}4 \\
- \\
-\end{array}$ & $\begin{array}{r}11 \\
- \\
-\end{array}$ & $\begin{array}{r}24 \\
5 \\
-\end{array}$ & $\begin{array}{r}46 \\
36 \\
-\end{array}$ & $\begin{array}{r}80 \\
159 \\
-\end{array}$ & $\begin{array}{r}130 \\
536 \\
25\end{array}$ & $\begin{array}{r}200 \\
1519 \\
260\end{array}$ & $\begin{array}{l}x^{2}+x y, y^{2}+x u \\
z^{2}+x u, z u+u^{2} \\
y z\end{array}$ & $H_{22}$ & $A_{22.1}$ \\
\hline 30 & $\begin{array}{l}1 \\
- \\
-\end{array}$ & $\begin{array}{l}4 \\
- \\
-\end{array}$ & $\begin{array}{r}11 \\
- \\
-\end{array}$ & $\begin{array}{r}25 \\
1 \\
-\end{array}$ & $\begin{array}{r}50 \\
9 \\
-\end{array}$ & $\begin{array}{r}91 \\
46 \\
-\end{array}$ & $\begin{array}{r}154 \\
175 \\
1\end{array}$ & $\begin{array}{r}246 \\
550 \\
14\end{array}$ & $\begin{array}{l}x y+u^{2}, x z \\
x^{2}+z^{2}+u^{2} \\
y^{2}, y u+z u\end{array}$ & $H_{23}$ & $A_{23.1}$ \\
\hline 31 & $\begin{array}{l}1 \\
- \\
-\end{array}$ & $\begin{array}{l}4 \\
- \\
-\end{array}$ & $\begin{array}{r}11 \\
- \\
-\end{array}$ & $\begin{array}{r}25 \\
2 \\
-\end{array}$ & $\begin{array}{r}50 \\
16 \\
-\end{array}$ & $\begin{array}{r}91 \\
77 \\
-\end{array}$ & $\begin{array}{r}154 \\
282 \\
4\end{array}$ & $\begin{array}{r}246 \\
864 \\
48\end{array}$ & $\begin{array}{l}x^{2}-y^{2}, y^{2}-z^{2} \\
z^{2}-u^{2}, x z+y u \\
-x^{2}+x y-y z+x u\end{array}$ & $H_{24}$ & $A_{23.1}$ \\
\hline 32 & $\begin{array}{l}1 \\
- \\
-\end{array}$ & $\begin{array}{l}4 \\
- \\
-\end{array}$ & $\begin{array}{r}11 \\
- \\
-\end{array}$ & $\begin{array}{r}25 \\
2 \\
-\end{array}$ & $\begin{array}{r}51 \\
15 \\
-\end{array}$ & $\begin{array}{r}97 \\
68 \\
-\end{array}$ & $\begin{array}{r}176 \\
238 \\
4 \\
\end{array}$ & $\begin{array}{r}309 \\
708 \\
44 \\
\end{array}$ & $\begin{array}{l}x^{2}+z^{2}, x z, \\
y^{2}, y u+z u, \\
u^{2}\end{array}$ & $H_{23}$ & $A_{23.2}$ \\
\hline 33 & $\begin{array}{l}1 \\
- \\
-\end{array}$ & $\begin{array}{l}4 \\
- \\
-\end{array}$ & $\begin{array}{r}11 \\
- \\
-\end{array}$ & $\begin{array}{r}25 \\
3 \\
-\end{array}$ & $\begin{array}{r}51 \\
22 \\
-\end{array}$ & $\begin{array}{r}97 \\
99 \\
-\end{array}$ & $\begin{array}{r}176 \\
345 \\
9\end{array}$ & $\begin{array}{r}309 \\
1024 \\
96\end{array}$ & $\begin{array}{l}x^{2}+x y, y^{2}+y z \\
y^{2}+x u, z^{2}+x u \\
z u+u^{2}\end{array}$ & $H_{24}$ & $A_{23.2}$ \\
\hline 34 & $\begin{array}{l}1 \\
-\end{array}$ & $\begin{array}{l}4 \\
-\end{array}$ & $\begin{array}{r}11 \\
-\end{array}$ & $\begin{array}{r}26 \\
-\end{array}$ & $\begin{array}{r}55 \\
-\end{array}$ & $\begin{array}{r}106 \\
5\end{array}$ & $\begin{array}{r}190 \\
38\end{array}$ & $\begin{array}{l}322 \\
172\end{array}$ & $\begin{array}{l}x^{2}+x y+y u+u^{2}, y^{2}, x z \\
x^{2}+z^{2}+u^{2}, y u+z u\end{array}$ & $H_{27}$ & $A_{27.1}$ \\
\hline 35 & $\begin{array}{l}1 \\
-\end{array}$ & $\begin{array}{l}4 \\
-\end{array}$ & $\begin{array}{r}11 \\
-\end{array}$ & $\begin{array}{r}26 \\
-\end{array}$ & $\begin{array}{r}55 \\
2\end{array}$ & $\begin{array}{r}108 \\
16\end{array}$ & $\begin{array}{r}201 \\
76\end{array}$ & $\begin{array}{l}360 \\
278\end{array}$ & $\begin{array}{l}x^{2}+z^{2}+u^{2}, y^{2}, x z \\
x y+y z+y u, y u+z u\end{array}$ & $H_{27}$ & $A_{27.2}$ \\
\hline 36 & $\begin{array}{l}1 \\
- \\
-\end{array}$ & $\begin{array}{l}4 \\
- \\
-\end{array}$ & $\begin{array}{r}11 \\
- \\
-\end{array}$ & $\begin{array}{r}26 \\
1 \\
-\end{array}$ & $\begin{array}{r}56 \\
8 \\
-\end{array}$ & $\begin{array}{r}114 \\
38 \\
-\end{array}$ & $\begin{array}{r}223 \\
140 \\
1 \\
\end{array}$ & $\begin{array}{r}424 \\
441 \\
12 \\
\end{array}$ & $\begin{array}{l}x^{2}+y^{2}, z^{2}, u^{2}, \\
y z-y u \\
x z+z u\end{array}$ & $H_{27}$ & $A_{27.3}$ \\
\hline 37 & $\begin{array}{l}1 \\
- \\
-\end{array}$ & $\begin{array}{l}4 \\
- \\
-\end{array}$ & $\begin{array}{r}11 \\
- \\
-\end{array}$ & $\begin{array}{r}26 \\
1 \\
-\end{array}$ & $\begin{array}{r}56 \\
8 \\
-\end{array}$ & $\begin{array}{r}114 \\
38 \\
-\end{array}$ & $\begin{array}{r}223 \\
141 \\
1\end{array}$ & $\begin{array}{r}425 \\
448 \\
12\end{array}$ & $\begin{array}{l}x^{2}, y^{2}, x y-z u \\
y z-x u \\
(x-y)(z-u)\end{array}$ & $H_{27}$ & $A_{27.4}$ \\
\hline
\end{tabular}


Table 5. Cases 38-57

\begin{tabular}{|c|c|c|c|c|c|c|c|c|c|c|c|}
\hline Case & \multicolumn{8}{|c|}{ The graded Betti Numbers } & ideal gen:s (ex.) & $R(t)$ & $R^{!}(t)$ \\
\hline 38 & 1 & 4 & 11 & 26 & 57 & 120 & 247 & 502 & $x^{2}, y^{2}, z^{2}, z u, u^{2}$ & $H_{25}$ & $A_{25}$ \\
\hline 39 & $\begin{array}{l}1 \\
- \\
-\end{array}$ & $\begin{array}{l}4 \\
- \\
- \\
\end{array}$ & $\begin{array}{r}11 \\
- \\
-\end{array}$ & $\begin{array}{r}26 \\
1 \\
-\end{array}$ & $\begin{array}{r}57 \\
7 \\
-\end{array}$ & $\begin{array}{r}120 \\
31 \\
-\end{array}$ & $\begin{array}{r}247 \\
109 \\
1 \\
\end{array}$ & $\begin{array}{r}502 \\
334 \\
10 \\
\end{array}$ & $\begin{array}{l}x^{2}+y z+u^{2}, \\
x z+z^{2}+y u, \\
x y, x u, z u\end{array}$ & $H_{26}$ & $A_{25}$ \\
\hline 40 & $\begin{array}{l}1 \\
- \\
-\end{array}$ & $\begin{array}{l}4 \\
- \\
-\end{array}$ & $\begin{array}{r}11 \\
- \\
-\end{array}$ & $\begin{array}{r}26 \\
2 \\
-\end{array}$ & $\begin{array}{r}57 \\
14 \\
-\end{array}$ & $\begin{array}{r}120 \\
62 \\
-\end{array}$ & $\begin{array}{r}247 \\
218 \\
4\end{array}$ & $\begin{array}{r}502 \\
668 \\
40\end{array}$ & $\begin{array}{l}x^{2}-x u, x u-y^{2} \\
y^{2}-z^{2}, z^{2}-u^{2} \\
x z+y u\end{array}$ & $H_{27}$ & $A_{25}$ \\
\hline 41 & 1 & 4 & 11 & 27 & 62 & 137 & 295 & 624 & $x y, y^{2}, z^{2}, z u, u^{2}$ & $H_{29}$ & $A_{29}$ \\
\hline 42 & $\begin{array}{l}1 \\
-\end{array}$ & $\begin{array}{l}4 \\
-\end{array}$ & $\begin{array}{r}11 \\
-\end{array}$ & $\begin{array}{r}27 \\
-\end{array}$ & $\begin{array}{r}62 \\
-\end{array}$ & $\begin{array}{r}137 \\
1\end{array}$ & $\begin{array}{r}296 \\
8\end{array}$ & $\begin{array}{r}632 \\
30\end{array}$ & $\begin{array}{l}x^{2}+x y, z u, y^{2} \\
x u, x z+y u\end{array}$ & $H_{29}$ & $A_{29.1}$ \\
\hline 43 & 1 & 4 & 11 & 27 & 63 & 144 & 326 & 735 & $x^{2}, y^{2}, y z, z u, u^{2}$ & $H_{28}$ & $A_{28}$ \\
\hline 44 & $\begin{array}{l}1 \\
- \\
-\end{array}$ & $\begin{array}{l}4 \\
- \\
-\end{array}$ & $\begin{array}{r}11 \\
- \\
-\end{array}$ & $\begin{array}{r}27 \\
1 \\
-\end{array}$ & $\begin{array}{r}63 \\
7 \\
-\end{array}$ & $\begin{array}{r}144 \\
31 \\
-\end{array}$ & $\begin{array}{r}326 \\
111 \\
1\end{array}$ & $\begin{array}{r}735 \\
352 \\
10\end{array}$ & $\begin{array}{l}x z, y z, y^{2} \\
y u+z u \\
z^{2}+u^{2}\end{array}$ & $H_{29}$ & $A_{28}$ \\
\hline 45 & 1 & 4 & 11 & 28 & 68 & 162 & 382 & 896 & $\begin{array}{l}x y+y z, x y+z^{2}+y u, \\
y u+z u, y^{2}, x z\end{array}$ & $H_{31}$ & $A_{31}$ \\
\hline 46 & 1 & 4 & 11 & 28 & 69 & 168 & 407 & 984 & $x^{2}, x y, y z, z u, u^{2}$ & $H_{30}$ & $A_{30}$ \\
\hline 47 & $\begin{array}{l}1 \\
- \\
-\end{array}$ & $\begin{array}{l}4 \\
- \\
-\end{array}$ & $\begin{array}{r}11 \\
- \\
-\end{array}$ & $\begin{array}{r}28 \\
1 \\
-\end{array}$ & $\begin{array}{r}69 \\
6 \\
-\end{array}$ & $\begin{array}{r}168 \\
25 \\
-\end{array}$ & $\begin{array}{r}407 \\
88 \\
1\end{array}$ & $\begin{array}{r}984 \\
282 \\
8\end{array}$ & $\begin{array}{l}x^{2}+x y, y^{2} \\
x u, x z+y u \\
-x^{2}+x z-y z\end{array}$ & $H_{31}$ & $A_{30}$ \\
\hline 48 & $\begin{array}{l}1 \\
- \\
-\end{array}$ & $\begin{array}{l}4 \\
- \\
-\end{array}$ & $\begin{array}{r}11 \\
- \\
-\end{array}$ & $\begin{array}{r}28 \\
1 \\
-\end{array}$ & $\begin{array}{r}69 \\
7 \\
-\end{array}$ & $\begin{array}{r}169 \\
31 \\
-\end{array}$ & $\begin{array}{r}413 \\
113 \\
1\end{array}$ & $\begin{array}{r}1009 \\
370 \\
10\end{array}$ & $\begin{array}{l}x y, z^{2}+y u \\
y u+z u, y^{2}, \\
x z\end{array}$ & $H_{31}$ & $A_{31.1}$ \\
\hline 49 & 1 & 4 & 11 & 29 & 75 & 193 & 496 & 1274 & $x z, y^{2}, z^{2}, y u, z u$ & $H_{33}$ & $A_{33}$ \\
\hline 50 & 1 & 4 & 11 & 29 & 76 & 199 & 521 & 1364 & $x^{2}, x y, x z, y^{2}, z^{2}$ & $H_{32}$ & $A_{32}$ \\
\hline 51 & $\begin{array}{l}1 \\
- \\
-\end{array}$ & $\begin{array}{l}4 \\
- \\
-\end{array}$ & $\begin{array}{r}11 \\
- \\
-\end{array}$ & $\begin{array}{r}29 \\
1 \\
-\end{array}$ & $\begin{array}{r}76 \\
6 \\
-\end{array}$ & $\begin{array}{r}199 \\
25 \\
-\end{array}$ & $\begin{array}{r}521 \\
90 \\
1\end{array}$ & $\begin{array}{r}1364 \\
300 \\
8\end{array}$ & $\begin{array}{l}x y, x z, y z+x u \\
z^{2}, z u\end{array}$ & $H_{33}$ & $A_{32}$ \\
\hline 52 & 1 & 4 & 11 & 30 & 82 & 224 & 612 & 1672 & $x^{2}, x y, x z, y^{2}, y z$ & $H_{34}$ & $A_{34}$ \\
\hline 53 & 1 & 4 & 11 & 31 & 88 & 249 & 705 & 1996 & $y^{2}-u^{2}, x z, y z, z^{2}, z u$ & $H_{35}$ & $A_{35}$ \\
\hline 54 & 1 & 4 & 12 & 32 & 80 & 192 & 448 & 1024 & $x^{2}, x z, y^{2}, z^{2}, y u+z u, u^{2}$ & $H_{13}$ & $A_{13}$ \\
\hline 55 & $\begin{array}{l}1 \\
- \\
\end{array}$ & $\begin{array}{l}4 \\
- \\
\end{array}$ & $\begin{array}{r}12 \\
- \\
\end{array}$ & $\begin{array}{r}32 \\
- \\
\end{array}$ & $\begin{array}{r}80 \\
- \\
\end{array}$ & $\begin{array}{r}192 \\
1 \\
\end{array}$ & $\begin{array}{r}449 \\
10 \\
\end{array}$ & $\begin{array}{r}1034 \\
57 \\
\end{array}$ & $\begin{array}{l}x^{2}+x y, x z+y u \\
x u, y^{2}, z^{2}, z u+u^{2}\end{array}$ & $H_{13}$ & $A_{13.1}$ \\
\hline 56 & $\begin{array}{l}1 \\
- \\
\end{array}$ & $\begin{array}{l}4 \\
- \\
\end{array}$ & $\begin{array}{r}12 \\
- \\
\end{array}$ & $\begin{array}{r}32 \\
- \\
\end{array}$ & $\begin{array}{r}80 \\
1 \\
\end{array}$ & $\begin{array}{r}193 \\
9 \\
\end{array}$ & $\begin{array}{r}457 \\
48 \\
\end{array}$ & $\begin{array}{r}1072 \\
199 \\
\end{array}$ & $\begin{array}{l}x^{2}+x z+u^{2}, x y \\
x u, x^{2}-y^{2}, z^{2}, z u\end{array}$ & $H_{13}$ & $A_{13.2}$ \\
\hline 57 & $\begin{array}{l}1 \\
- \\
-\end{array}$ & $\begin{array}{l}4 \\
- \\
-\end{array}$ & $\begin{array}{r}12 \\
- \\
-\end{array}$ & $\begin{array}{r}32 \\
1 \\
-\end{array}$ & $\begin{array}{r}81 \\
8 \\
-\end{array}$ & $\begin{array}{r}200 \\
40 \\
-\end{array}$ & $\begin{array}{r}488 \\
160 \\
1\end{array}$ & $\begin{array}{r}1184 \\
562 \\
12\end{array}$ & $\begin{array}{l}x^{2}+y z+u^{2}, x u \\
x^{2}+x y, x z+y u \\
z u+u^{2}, y^{2}+z^{2}\end{array}$ & $H_{13}$ & $A_{13.3}$ \\
\hline
\end{tabular}


Table 6. Cases 58-74

\begin{tabular}{|c|c|c|c|c|c|c|c|c|c|c|c|}
\hline Case & \multicolumn{8}{|c|}{ The graded Betti Numbers } & ideal gen:s,ex. & $R(t)$ & $R^{!}(t)$ \\
\hline 58 & 1 & 4 & 12 & 33 & 87 & 225 & 576 & 1467 & $\begin{array}{l}x^{2}+x y, x^{2}+z u, y^{2} \\
z^{2}, x z+y u, x u\end{array}$ & $H_{15}$ & $A_{15}$ \\
\hline 59 & $\begin{array}{l}1 \\
-\end{array}$ & $\begin{array}{l}4 \\
-\end{array}$ & $\begin{array}{r}12 \\
-\end{array}$ & $\begin{array}{r}33 \\
-\end{array}$ & $\begin{array}{r}87 \\
-\end{array}$ & $\begin{array}{r}225 \\
-\end{array}$ & $\begin{array}{r}576 \\
1\end{array}$ & $\begin{array}{r}1468 \\
8\end{array}$ & $\begin{array}{l}x^{2}-y^{2}, x y, x u \\
z^{2}, z u, x z+y u\end{array}$ & $H_{15}$ & $A_{15.1}$ \\
\hline 60 & $\begin{array}{l}1 \\
-\end{array}$ & $\begin{array}{l}4 \\
-\end{array}$ & $\begin{array}{r}12 \\
-\end{array}$ & $\begin{array}{r}33 \\
-\end{array}$ & $\begin{array}{r}87 \\
-\end{array}$ & $\begin{array}{r}225 \\
1\end{array}$ & $\begin{array}{r}577 \\
7\end{array}$ & $\begin{array}{r}1474 \\
33\end{array}$ & $\begin{array}{l}x^{2}+y z+u^{2}, x z+y u, \\
z u, x^{2}+x y, z^{2}, x u\end{array}$ & $H_{15}$ & $A_{15.2}$ \\
\hline 61 & 1 & 4 & 12 & 33 & 88 & 232 & 609 & 1596 & $\begin{array}{l}x^{2}-y^{2}, x y, z^{2}, \\
x u, z u, u^{2}\end{array}$ & $H_{14}$ & $A_{14}$ \\
\hline 62 & $\begin{array}{l}1 \\
- \\
-\end{array}$ & $\begin{array}{l}4 \\
- \\
-\end{array}$ & $\begin{array}{r}12 \\
- \\
-\end{array}$ & $\begin{array}{r}33 \\
1 \\
-\end{array}$ & $\begin{array}{r}88 \\
7 \\
-\end{array}$ & $\begin{array}{r}232 \\
33 \\
-\end{array}$ & $\begin{array}{r}609 \\
129 \\
1\end{array}$ & $\begin{array}{r}1596 \\
455 \\
10\end{array}$ & $\begin{array}{l}x^{2}-y^{2}, x y \\
x u, y z+y u \\
z^{2}, z u\end{array}$ & $H_{15}$ & $A_{14}$ \\
\hline 63 & 1 & 4 & 12 & 34 & 94 & 258 & 706 & 1930 & $x^{2}, x y, x u, y^{2}, z^{2}, z u$ & $H_{17}$ & $A_{17}$ \\
\hline 64 & 1 & 4 & 12 & 34 & 95 & 265 & 739 & 2061 & $\begin{array}{l}x^{2}-y^{2}, x y \\
z^{2}, x u, y u, z u\end{array}$ & $H_{16}$ & $A_{16}$ \\
\hline 65 & $\begin{array}{l}1 \\
- \\
-\end{array}$ & $\begin{array}{l}4 \\
- \\
-\end{array}$ & $\begin{array}{r}12 \\
- \\
-\end{array}$ & $\begin{array}{r}34 \\
1 \\
-\end{array}$ & $\begin{array}{r}95 \\
7 \\
-\end{array}$ & $\begin{array}{r}265 \\
33 \\
-\end{array}$ & $\begin{array}{r}739 \\
131 \\
1\end{array}$ & $\begin{array}{r}2061 \\
475 \\
10\end{array}$ & $\begin{array}{l}x^{2}, x y, x z \\
y^{2}, y u+z^{2} \\
y u+z u\end{array}$ & $H_{17}$ & $A_{16}$ \\
\hline 66 & 1 & 4 & 12 & 35 & 101 & 291 & 838 & 2413 & $x z, y^{2}, y u, z^{2}, z u, u^{2}$ & $H_{18}$ & $A_{18}$ \\
\hline 67 & 1 & 4 & 12 & 36 & 107 & 318 & 945 & 2808 & $x y, x z, y^{2}, y u, z^{2}, z u$ & $H_{20}$ & $A_{20}$ \\
\hline 68 & 1 & 4 & 12 & 36 & 108 & 324 & 972 & 2916 & $x^{2}, x y, x z, y^{2}, y z, z^{2}$ & $H_{19}$ & $A_{19}$ \\
\hline 69 & $\begin{array}{l}1 \\
- \\
-\end{array}$ & $\begin{array}{l}4 \\
- \\
-\end{array}$ & $\begin{array}{r}12 \\
- \\
-\end{array}$ & $\begin{array}{r}36 \\
1 \\
-\end{array}$ & $\begin{array}{r}108 \\
6 \\
-\end{array}$ & $\begin{array}{r}324 \\
27 \\
-\end{array}$ & $\begin{array}{r}972 \\
108 \\
1\end{array}$ & $\begin{array}{r}2916 \\
405 \\
8\end{array}$ & $\begin{array}{l}x^{2}, x z, x u \\
x y-z u \\
y z, z^{2}\end{array}$ & $H_{20}$ & $A_{19}$ \\
\hline 70 & 1 & 4 & 12 & 37 & 114 & 351 & 1081 & 3329 & $x^{2}, x y, x z, x u, y^{2}, y z$ & $H_{21}$ & $A_{21}$ \\
\hline 71 & 1 & 4 & 13 & 40 & 121 & 364 & 1093 & 3280 & $\begin{array}{l}x^{2}, y^{2}, z^{2}, u^{2}, \\
x y, z u, y z+x u\end{array}$ & $H_{7}$ & $A_{7}$ \\
\hline 72 & 1 & 4 & 13 & 41 & 128 & 399 & 1243 & 3872 & $\begin{array}{l}x^{2}-y^{2}, x y, y z \\
z u, z^{2}, x z+y u, x u\end{array}$ & $H_{9}$ & $A_{9}$ \\
\hline 73 & 1 & 4 & 13 & 41 & 129 & 406 & 1278 & 4023 & $\begin{array}{l}x^{2}, y^{2}, z^{2}, u^{2}, \\
z u, y u, x u\end{array}$ & $H_{8}$ & $A_{8}$ \\
\hline 74 & $\begin{array}{l}1 \\
- \\
-\end{array}$ & $\begin{array}{l}4 \\
- \\
-\end{array}$ & $\begin{array}{r}13 \\
- \\
-\end{array}$ & $\begin{array}{r}41 \\
1 \\
-\end{array}$ & $\begin{array}{r}129 \\
7 \\
-\end{array}$ & $\begin{array}{r}406 \\
35 \\
-\end{array}$ & $\begin{array}{r}1278 \\
151 \\
1\end{array}$ & $\begin{array}{r}4023 \\
604 \\
10\end{array}$ & $\begin{array}{l}x^{2}, x y+z^{2} \\
y z, x u, y u \\
z u, u^{2}\end{array}$ & $H_{9}$ & $A_{8}$ \\
\hline
\end{tabular}


Table 7. Cases 75-83

\begin{tabular}{|c|c|c|c|c|c|c|c|c|c|c|c|}
\hline Case & \multicolumn{8}{|c|}{ The graded Betti Numbers } & ideal gen:s & $R(t)$ & $R^{!}(t)$ \\
\hline 75 & 1 & 4 & 13 & 42 & 135 & 434 & 1395 & 4484 & $\begin{array}{l}x^{2}, x y, x z, x u \\
y^{2}, y z, u^{2}\end{array}$ & $H_{10}$ & $A_{10}$ \\
\hline 76 & 1 & 4 & 13 & 43 & 142 & 469 & 1549 & 5116 & $\begin{array}{l}x^{2}, x y, x z, x u \\
z^{2}, z u, y u\end{array}$ & $H_{11}$ & $A_{11}$ \\
\hline 77 & 1 & 4 & 13 & 44 & 148 & 498 & 1676 & 5640 & $\begin{array}{l}x^{2}, x y, x z, x u, \\
y^{2}, y z, y u\end{array}$ & $H_{12}$ & $A_{12}$ \\
\hline 78 & 1 & 4 & 14 & 48 & 164 & 560 & 1912 & 6528 & $\begin{array}{l}x^{2}, x y, y^{2}, z^{2}, z u \\
u^{2}, x z+y u, y z-x u\end{array}$ & $H_{4}$ & $A_{4}$ \\
\hline 79 & 1 & 4 & 14 & 49 & 171 & 597 & 2084 & 7275 & $\begin{array}{l}x^{2}, x y, x z, x u \\
y^{2}, y u, z^{2}, z u\end{array}$ & $H_{5}$ & $A_{5}$ \\
\hline 80 & 1 & 4 & 14 & 50 & 178 & 634 & 2258 & 8042 & $\begin{array}{l}x^{2}, x y, x z, y^{2} \\
y z, y u, z^{2}, z u\end{array}$ & $H_{6}$ & $A_{6}$ \\
\hline 81 & 1 & 4 & 15 & 56 & 209 & 780 & 2911 & 10864 & $\begin{array}{l}x^{2}, y^{2}, z^{2}, u^{2}, x y \\
x z, y z-x u, y u, z u\end{array}$ & $H_{2}$ & $A_{2}$ \\
\hline 82 & 1 & 4 & 15 & 57 & 216 & 819 & 3105 & 11772 & $\begin{array}{l}x^{2}, x y, x z, x u, y^{2}, \\
z u, u^{2}, y z, y u\end{array}$ & $H_{3}$ & $A_{3}$ \\
\hline 83 & 1 & 4 & 16 & 64 & 256 & 1024 & 4096 & 16384 & $\begin{array}{l}x^{2}, y^{2}, z^{2}, u^{2}, x y, \\
x z, x u, y z, y u, z u\end{array}$ & $H_{1}$ & $A_{1}$ \\
\hline
\end{tabular}


Table 8 . The 16 series $R^{!}(t)$ that do not correspond to Koszul algebras.

\begin{tabular}{|r|l|l|l|}
\hline Rings & $R^{!}(t)$ & Numerator of $R^{!}(t)$ & Denominator of $R^{!}(t)$ \\
\hline 17 & $A_{43.1}$ & $(1+t)^{4}\left(1+t^{3}\right)$ & $\left(1-t^{2}\right)^{4}$ \\
\hline 22 & $A_{46.1}$ & $(1+t)^{2}\left(1-t+t^{2}\right)$ & $(1-t)^{2}\left(1-t-t^{2}-t^{4}-t^{5}\right)$ \\
\hline 29 & $A_{22.1}$ & $(1+t)^{4}$ & $\left(1-t^{2}\right)^{5}$ \\
\hline 30,31 & $A_{23.1}$ & $(1+t)^{4}\left(1+t^{3}\right)$ & $\left(1-t^{2}\right)^{5}$ \\
\hline 32,33 & $A_{23.2}$ & $(1+t)^{3}$ & $\left(1-t^{2}\right)^{3}\left(1-t-t^{2}\right)$ \\
\hline 34 & $A_{27.1}$ & $(1+t)^{4}\left(1+t^{3}\right)^{2}$ & $\left(1-t^{2}\right)^{5}\left(1-t^{4}\right)$ \\
\hline 35 & $A_{27.2}$ & $(1+t)^{3}\left(1+t^{3}\right)$ & $\left(1-t^{2}\right)^{3}\left(1-t-t^{2}\right)$ \\
\hline 36 & $A_{27.3}$ & $(1+t)^{2}$ & $\left(1-t^{2}\right)\left(1-t-t^{2}\right)^{2}$ \\
\hline 37 & $A_{27.4}$ & $(1+t)^{3}\left(1+t^{3}\right)$ & $\left(1-t^{2}\right)^{3}\left(1-t-t^{2}-t^{4}-t^{5}\right)$ \\
\hline 42 & $A_{29.1}$ & $(1+t)^{2}\left(1+t^{3}\right)$ & $\left(1-t^{2}\right)^{2}\left(1-2 t-t^{4}\right)$ \\
\hline 48 & $A_{31.1}$ & $(1+t)^{2}$ & $1-2 t-2 t^{2}+2 t^{3}+t^{4}-t^{5}$ \\
\hline 55 & $A_{13.1}$ & $\left(1-t+t^{2}\right)^{2}$ & $(1-t)^{3}\left(1-3 t+3 t^{2}-3 t^{3}\right)$ \\
\hline 56 & $A_{13.2}$ & $\left(1-t+t^{2}\right)$ & $(1-t)^{2}\left(1-3 t+2 t^{2}-t^{3}\right)$ \\
\hline 57 & $A_{13.3}$ & 1 & $(1-t)^{2}\left(1-2 t-t^{2}\right)$ \\
\hline 59 & $A_{15.1}$ & $\left(1+t^{3}\right)$ & $(1-t)^{2}\left(1-2 t-t^{2}-2 t^{4}-t^{5}\right)$ \\
\hline 60 & $A_{15.2}$ & 1 & $(1-t)^{2}\left(1-2 t-t^{2}-t^{3}\right)$ \\
\hline
\end{tabular}

For e.g. the ring 34 the nilpotency degree of $\eta$ is 5 . 